\title{
LOS CARGOS: UN RELATO DEL SISTEMA DE CARGOS EN EL TOTONACO DEL RÍO NECAXA
}

\section{LOS CARGOS: AN ACCOUNT OF THE SYSTEM OF CARGOS IN UPPER NECAXA TOTONAC}

\author{
DAVID BECK \\ YVONNE LAM
}

Narración: ROSENDO MELO MÁRQuEZ

\begin{abstract}
Resumen. En este artículo se presenta un texto del totonaco del Río Necaxa en el cual se describe el ciclo ceremonial anual para asignar y renovar los puestos municipales, o sea, los cargos. El texto ofrece un relato de un género muy poco documentado: la descripción de primera mano de los festivales y los rituales en torno al sistema de cargos. Les infunde vida a los trabajos académicos anteriores acerca del sistema mesoamericano de cargos al describir una imagen clara y detallada de cómo se practicaba a mediados del siglo xx en la Sierra Norte de Puebla. PaLABRAS ClavE: totonaco, cargos, Mesoamérica, administración cívico-religiosa, ciclos ceremoniales.
\end{abstract}

Summary. In this article, we present a text in Upper Necaxa Totonac describing the annual ceremonial cycle of the assignment and renewal of municipal offices, or cargos. This text provides a rare first-person account of the festivals and rituals surrounding the system of cargos, breathing some life into earlier academic accounts of the Mesoamerican cargo system and painting a clear and detailed picture of the system as it was practiced in the mid-twentieth century in the Sierra Norte de Puebla.

KEYWORDS: Totonac, cargos, Mesoamerica, civil-religious administration, ceremonial cycles.

\section{Introducción ${ }^{1}$}

Aunque los lingüistas que colaboran con las comunidades indígenas se han dedicado tradicionalmente a recoger mitos, leyendas, cuentos tradicionales y otros estilos narrativos, han sido principalmente los antropólogos quienes se han preocupado por la documentación de las prácticas culturales y las formas de organización social. Sin embargo, no se han interesado mucho en registrarlas a través de los textos contados por las personas que participan en ellas, de ahí la relativa escasez de narraciones de primera mano sobre eventos sociales y públicos, como fiestas y ciclos ceremoniales, donde resaltan el idioma y los usos lingüísti-

${ }^{1}$ Deseamos agradecer a Rosendo Melo Márquez por contarnos el relato, así como a Longino Barragán Sampayo y a Porfirio Sampayo Macín por su ayuda en la transcripción y la traducción del texto. También agradecemos a Paulette Levy, a Sara Raquel Baltazar Rangel y a los dictaminadores anónimos y los editores de Tlalocan por sus observaciones y sugerencias. 
cos asociados a estas prácticas culturales. En este artículo se presenta un relato narrado por don Rosendo Melo Márquez, hablante nativo del totonaco del Río Necaxa [iso tku], en el cual describe el ciclo ceremonial que tenía lugar cada año para asignar y renovar los puestos municipales, es decir, los cargos, y reflexiona sobre su pérdida en la época actual. La exposición de don Rosendo da vida a lo que ya sabemos sobre el sistema mesoamericano de cargos en los trabajos académicos (p. ej., Cancian, 1965; Dewalt, 1975; Friedlander, 1981; Chance y Taylor, 1985; Caulfield et al., 2005; Masferrer Kan, 2006; Stresser-Péan, 2009). Este relato, aunado a los datos recolectados en entrevistas hechas posteriormente con otros habitantes de Patla y Chicontla, en particular con don Longino Barragán Sampayo, nos ofrece una imagen clara y detallada del sistema de cargos tal como se practicaba a mediados del siglo xx en la Sierra Norte de Puebla.

\section{El sistema de cargos}

El sistema de cargos era una estructura administrativa cívico-religiosa que se encontraba en comunidades indígenas por toda Mesoamérica (Chance y Taylor, 1985). Muchos creen que tiene su origen en el sistema de administración civil que fue impuesto por las autoridades coloniales sobre las comunidades indígenas en el centro y el sur de México durante los años que siguieron a la invasión y la conquista española (Masferrer Kan, 2006; Stresser-Péan, 2009). Aunque incorporó algunos elementos prehispánicos (Carrasco, 1982), se basó principalmente en las estructuras administrativas de España que incluían a alcaldes, regidores y otros cargos municipales del Viejo Mundo (Chance y Taylor, 1985). En la Sierra Norte de Puebla la reorganización de las comunidades originarias según el nuevo sistema se implementó en una época relativamente tardía, como parte de la evangelización católica de la región a finales del siglo XvI (Stresser-Péan, 2009: 59).

This organization progressively replaced the government of the hereditary cacique, descendant of the ancient lords of the past. The civil organization was duplicated by a religious organization with chanters, sextons, catechists, bell ringers, and grave diggers, among others, directed by a fiscal (Stresser-Péan, 2009: 60).

${ }^{2}$ El totonaco del Río Necaxa es una lengua de la rama totonaca de la familia lingüística totonaco-tepehua. Lo hablan unas 3000 personas de cuatro pueblos de la Sierra Norte de Puebla: Patla, Chicontla, Cacahuatlán y San Pedro Tlalontongo (INEGI 2010; Beck, 2011). 
Esta organización reemplazó poco a poco al gobierno del cacique hereditario, descendiente de los antiguos señores del pasado. La organización civil fue duplicada por una organización religiosa compuesta de cantores, sacristanes, catequistas, campaneros y sepultureros, entre otros, bajo la dirección de un fiscal (traducción de los autores).

En el Valle del Necaxa, las autoridades religiosas, bajo la dirección de los fiscales de rango superior, cuidaban la iglesia en nombre del cura párroco, quien no vivía en la región hasta recientemente. Los fiscales se encargaban de organizar las festividades y de pagar por ellas, incluyendo la fiesta patronal y las celebraciones asociadas con el sistema de cargos que se describen en el texto que sigue. Las autoridades municipales se ocupaban de las responsabilidades normales del gobierno municipal, siendo tal vez la más importante (a juzgar por los recuerdos de nuestros colaboradores) la organización de las faenas, es decir, el trabajo comunal. Estas consistían en actividades semanales en las que todos los hombres en buena condición física estaban obligados a participar para llevar a cabo diversas obras públicas, como la construcción y el mantenimiento de los caminos, la iglesia, el ayuntamiento (la presidencia) y, más tarde, las escuelas. Don Longino con frecuencia recuerda haber trabajado en la edificación de la primera escuela primaria de Chicontla a mediados de la década de 1970, construcción que fue posible gracias a los donativos (cooperaciones) de la comunidad bajo la iniciativa del alcalde, otro de nuestros colaboradores, el difunto Luciano Cabrera Trinidad. Las obras que llevarían a cabo los faeneros eran seleccionadas con antelación por el alcalde y los regidores. Una noche antes de la faena, los ma:skuju:nu'nín 'avisadores' pasaban por el pueblo gritando "Ihe: $n$ " para avisarle a la gente que se reunirían en la presidencia a la mañana siguiente. A quienes llegaban tarde o que no se presentaban, los multaban o los encarcelaban. Según don Longino y otros habitantes, la economía basada en el dinero que acompañó al cultivo del café contribuyó a la disminución de la participación en las faenas porque les permitió a muchos pagar la multa para no participar.

Para un joven, el sistema de cargos representaba una secuencia ascendente de roles sociales, cada uno con mayor prestigio, que desempeñaría a lo largo de su vida (Stresser-Péan, 2009: 61). En Patla y en Chicontla las personas de mayor edad hablan con frecuencia de los cargos que tuvieron en el pasado y se acuerdan de los difuntos de la comunidad, como don Luciano, relacionándolos con los puestos más altos que lograron durante su vida. Según Stresser-Péan, por toda la Sierra Norte

[t]he highest ranks of the hierarchy were held by a small number of individuals who had the greatest authority and enjoyed maximum prestige. Fulfilling these offices entailed the costs of sponsoring festivals, dances, and banquets, which were part of the normal routine in an indi- 
genous community. In the cities and more important villages, only the rich could reach the top of the social pyramid. They could not succeed except by committing part of their fortune to the community (Stresser-Péan, 2009: 61).

[1]os rangos más altos de la jerarquía eran ocupados por un número limitado de individuos que poseía mayor autoridad y gozaba del máximo prestigio. El desempeño de estos cargos involucraba los costos para patrocinar fiestas, bailes y comidas, una parte de la rutina normal dentro de una comunidad indígena. En las ciudades y en los pueblos de mayor importancia, sólo las personas adineradas eran capaces de llegar a la cumbre de la pirámide social. No podían tener éxito más que dedicando una parte de su fortuna a la comunidad (traducción de los autores).

En Patla y en Chicontla muchos hablan de los gastos que implicaba el ocupar un puesto, en particular la contratación de peones porque resultaba imposible cuidar su propio terreno debido a los deberes del cargo. En efecto, varios colaboradores atribuyen la disolución del sistema de cargos, y del sistema de faenas correspondiente, a la deuda que se contraía por participar en ello. Sin embargo, otras personas, incluido don Rosendo, hicieron hincapié en el aumento del contacto con el exterior como causa de la pérdida concurrente de la cultura y de la lengua.

Se ha documentado un sistema de cargos como el descrito por nuestros colaboradores en la zona de la Sierra Norte en general (Masferrer Kan, 2006; Stresser-Péan, 2009) y parece que se mantiene todavía en algunas comunidades del sureste (Adela Juárez, comunicación personal). Sin embargo, en el Valle del Necaxa, parece haber llegado a su fin en las décadas de 1970 y 1980, a medida que disminuía la participación popular en los festivales. Durante esta época, empezó a llegar a la región una mayor afluencia de extraños, incluidos misioneros protestantes, y aumentó la comunicación con la sociedad nacional mexicana, debido en parte a la creciente dependencia económica de la producción del café. En 1998, la primera visita de David Beck a la región, ya no quedaba ningún elemento del sistema salvo una faena semanal, cada vez menos frecuente y más irregular al pasar los años, hasta desaparecer completamente en Chicontla a finales de la década de 2000. En Patla todavía hay faenas de vez en cuando, convocadas para fines extraordinarias, pero pocos asisten y el trabajo comunal ha sido reemplazado casi totalmente por el trabajo pagado por el gobierno municipal. Todavía existen los cargos de alcalde y de regidor, pero por lo visto son iguales en esencia a los que existen en cualquier pueblo mexicano y parecen haberse integrado completamente al sistema nacional de partidos políticos. El sistema de fiscales ya no existía en ninguna de las dos comunidades cuando llegó Beck por primera vez. 
Los cargos

Los cargos tradicionales de Patla y Chicontla eran los siguientes:

albkalhtéj 'alcalde/presidente municipal' (del español alcalde) funcionario público superior, elegido por votación popular;

bo:lu:pixká:lb 'viejo fiscal' (ho:lú' 'viejo+ pixká:lh 'fiscal') fiscal de rango superior, generalmente un hombre mayor, encargado de los otros fiscales, puede que hubiera uno o dos de ellos;

laxi'tú:Ib auxiliar del alcalde encargado de desplumar los pollos para las comidas, en general había dos de ellos;

ma:skuju:nú' 'avisador' (ma:- 'cs' + skuj- 'trabajar' + -u: 'CS' + -nú' 'AGT') funcionario encargado de organizar a los trabajadores para la faena, había entre diez y quince de ellos, cada uno responsable de anunciar la faena a una manzana particular del pueblo;

ma:yú:lb 'topil' (del español mayor, posiblemente forma reducida de mayordomo) auxiliar del alcalde encargado de vigilar la comunidad y realizar actos oficiales, había entre diez y quince de ellos, cada uno asignado a una manzana particular del pueblo;

ma:yu:Ihpixká:lb 'fiscal mayor' (del español mayor + fiscal) fiscal de rango medio;

makastankú' nu' 'suplente' (maka- 'mano' + stánku' 'hermano menor' + -nu' 'PL') miembros de un grupo de cinco funcionarios municipales, se le asignaba a cada uno trabajar para el alcalde o para uno de los regidores (también xastankúnu' skujnín);

pixká:lb 'fiscal' (del español fiscal) funcionario laico de la iglesia encargado de las fiestas y del mantenimiento de la iglesia y de la residencia del cura, había de seis a ocho de ellos, divididos en tres rangos;

pixka:lhteniénte 'fiscal teniente' (del español fiscal + teniente) fiscal de rango inferior;

pu:la:siyáj 'policía' (del español policía) agente de policía, bajo la dirección del alcalde y de los regidores;

pu:'la:ná' 'comité asesor' (pu:'lá 'dirigir' + -ná' 'AGT') comité asesor para el alcalde que consistía en diez a quince hombres de rango superior;

puxku'ma:skuju:nú' 'jefe de los avisadores' (púxku' 'hermano mayor' + ma:skuju:nú' 'avisador') jefe de los funcionarios encargados de organizar a los trabajadores para la faena (ma:skuju:nú');

puxku'ma:yú:lb 'jefe de los topiles' (púxku' 'hermano mayor' + ma:yú:lh 'topil') jefe de los topiles (ma:yú:lh); ${ }^{3}$

${ }^{3}$ Anotar que los últimos dos términos incorporan el término de parentesco púxku' 'hermano mayor' para referirse al miembro de rango más alto de cada grupo (avisadores y topiles). El término púxku' por sí solo se emplea con frecuencia para señalar 'jefe' y en algunas partes del texto (p. ej., línea 37) se ve su uso para referirse al grupo de funcionarios municipales de rango superior, de la misma forma que se emplea la palabra correspondiente stánku' 'hermano menor' para indicar a los funcionarios de rango inferior (p. ej., línea 38). 
skújni' 'regidor' (skuj- 'trabajar' + -ni' 'NM') funcionario público que trabaja directamente bajo la dirección del alcalde, generalmente había cuatro de ellos: uno para la gobernación y la seguridad, uno para las obras públicas, uno para la educación y uno para la salud pública.

Según don Longino, la estructura de gobierno de estos cargos era como aparece en la figura 1, siendo el puesto más alto el del albkalbtéj 'alcalde', quien aceptaba consejos de un comité asesor (pu:'la:ná'). Los cargos de rango inferior eran ocupados por los hombres más jóvenes y los escalones más bajos eran para los muchachos, ello coincide con las observaciones hechas por Stresser-Péan (2009) en otras comunidades de la Sierra Norte. Los puestos superiores, incluido el comité asesor, se reservaban para los que tenían mayor estatus social y que tenían suficientes recursos económicos para pagar los gastos asociados con el desempeño de un cargo alto.

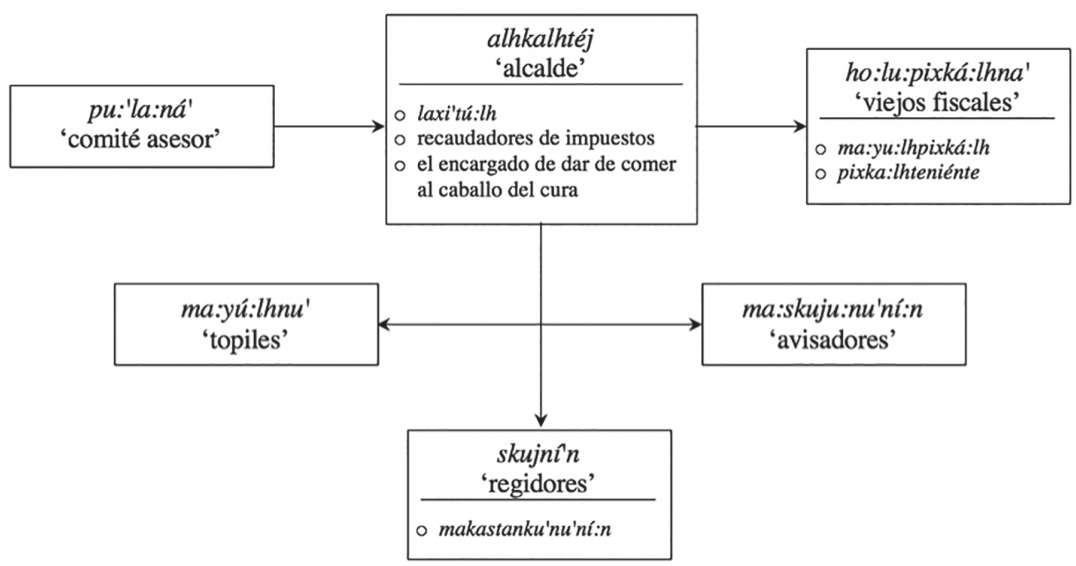

Figura 1: Estructura de gobernación tradicional

Es importante hacer notar que, de acuerdo al diagrama, don Longino considera que los fiscales eran subordinados, en cierto sentido, al alcalde como jefe de las autoridades civiles, lo que puede ser reflejo de la observación de Stresser-Péan (2009: 58) de que los alcaldes y los regidores administraban el apoyo financiero que recibían los fiscales de la comunidad. Sin embargo, en el relato de don Rosendo se observa una diferencia nominal en el equilibro de poder, dado que la distribución ceremonial de los cargos era la responsabilidad de los 
fiscales o las autoridades religiosas. En realidad, con excepción del cargo electo del alcalde, los puestos para el año entrante se decidían durante reuniones dirigidas por los funcionarios en el poder, principalmente el comité asesor (pu:'la:ná'). Por lo tanto, al menos en la época moderna, el papel de mando de los fiscales en las ceremonias para el cambio de cargos era solamente ceremonial, quizás un vestigio de la legitimación de la autoridad civil por parte de la Iglesia. Masferrer Kan (2006: 308) considera que en la Sierra Norte, el establecimiento de sistemas de cargos que combinaban la religión y la política representaba un intento deliberado por parte de las autoridades coloniales de socavar los puestos hereditarios tradicionales y los cacicazgos de antes de la conquista, y una manera de reforzar este intento consistía en hacer los puestos directamente subordinados a la autoridad de la Iglesia. Por otro lado, Chance y Taylor (1985) sostienen que la incorporación de las jerarquías laicas religiosas al sistema de cargos fue un cambio relativamente reciente que data del siglo xIx. A su parecer, la integración de las cofradías al sistema sería el resultado de una necesidad creciente de recabar recursos privados para el financiamiento de las fiestas públicas. En su narración, don Rosendo alude a una asociación entre la renovación de los cargos administrativos municipales y la procesión de la Virgen, lo que parece indicar un vínculo entre las cofradías y el sistema de cargos en Patla y en Chicontla.

\section{Convenciones empleadas en el texto}

El texto se presenta en formato interlineal con cinco líneas: la primera representa una transcripción de lo dicho en la ortografía práctica; la segunda indica el corte morfémico; la tercera consiste en la glosa por morfos; y la cuarta y la quinta líneas muestran la traducción libre en español y en inglés, respectivamente. La división en líneas refleja mayormente las intuiciones de los transcriptores con respecto a dónde ocurren las pausas naturales; parece representar tanto los enunciados "completos" como las pausas más largas entre enunciados. Con sólo una o dos excepciones, se ha hecho caso a las decisiones sobre la división en líneas que se tomaron al momento de la transcripción. Las únicas excepciones son contextos donde la división habría dificultado la comprensión del significado.

La ortografía que se emplea en las primeras dos líneas sigue el sistema elaborado para el totonaco del Río Necaxa en el Upper Necaxa Totonac Dictionary (Beck, 2011). Está de acuerdo con las convenciones comunes de las ortografías prácticas que se usan en muchas partes de Mesoamérica, las que, a su vez, se basan principalmente en las convenciones estándares de la ortografía española, con las siguientes diferencias: el uso de $<\mathrm{k}>\mathrm{para} / \mathrm{k} /$ (en vez del $<$ c/qu $>$ español), $<\mathrm{h}>$ para $/ \mathrm{R} /,<\mathrm{x}>$ para $/ \mathrm{J} /,<\mathrm{tz}>$ para $/ \mathrm{ts} /,<\mathrm{lh}>$ para $/ \mathrm{t} / \mathrm{y}<\mathrm{nh}>$ 
para /y/. Los dos puntos $<$ : > representan la duración de la vocal, el apóstrofo recto $<$ ' $>$ indica la laringización de la vocal precedente y la comilla simple $<$ ' > después de un fricativo indica la glotalización de la consonante. En la primera línea, el acento agudo (la tilde) marca el acento tónico léxico, que es último cuando la sílaba final es pesada (termina en consonante o en vocal larga) y es penúltimo cuando la palabra termina en vocal corta. La excepción son las palabras que terminan en algunos sufijos y enclíticos que tienen un acento inherente, es decir, un acento no predecible por su forma fonológica. En la segunda línea, la tilde señala tanto estos acentos inherentes como los acentos predecibles que tendrían las raíces y los sufijos polisilábicos. Los préstamos no naturalizados se escriben con letra cursiva en la primera línea según la ortografía española. Los falsos comienzos y los errores de habla obvios se encierran entre corchetes ([ ]); estos se incluyen en la transcripción de la primera línea cuando fueron lo suficientemente sobresalientes como para que los escribieran los transcriptores, pero no se incluyen en el análisis de la segunda y la tercera línea. Las pausas largas y las frases sin terminar se indican mediante puntos suspensivos (...).

La segunda y la tercera línea presentan el análisis por morfos. Los morfos y los lexemas de la segunda línea se escriben en su forma más completa sin recurrir a la etimología. El nivel de análisis en estas líneas trata de representar lo que es claramente una morfología productiva sin dividir demasiados elementos en sus componentes etimológicos, aunque incluimos un poco de lo último donde opinamos que la segmentación ayuda a dar una idea de los procesos típicos de la formación de palabras en el idioma. Los términos gramaticales no se definen aquí, pero siguen el uso de Beck $(2004,2011)$. Las abreviaturas se presentan después del texto al final del artículo.

Las traducciones al español y al inglés reflejan la expresión más idiomática del significado que tiene mayor semejanza con la estructura del enunciado original. Puesto que la información estructural se puede recuperar en gran parte del análisis, en aquellos lugares donde la estructura al nivel de la frase y la idiomaticidad estaban en conflicto se decidió a favor de la última. No obstante, las traducciones reflejan el tiempo verbal de las frases totonacas aun cuando los narradores anglohablantes o hispanohablantes habrían optado por otras formas. Cabe señalar que en muchos casos las traducciones son bastante distintas de las que ofreció don Rosendo durante las primeras sesiones de transcripción, que eran con frecuencia más una explicación de los eventos descritos que una traducción. La mayoría de las traducciones resultan de discusiones sobre frases particulares que se hicieron posteriormente con varias personas diferentes y también de un análisis minucioso de las glosas interlineales. 


\section{El texto}

Este texto es una narración de Rosendo Melo Márquez, quien nació en Patla en 1948. Los eventos que relata representan sus recuerdos de cómo se asignaban los cargos y cómo se celebraban la Navidad, el Año Nuevo, el Carnaval y la Pascua en la década de 1970 cuando era joven. Don Rosendo dice que él mismo tuvo un cargo en varias ocasiones, probablemente uno de los puestos de rango inferior como makastankú'nu' 'suplente' o ma:skuju:nú' 'avisador', pero su participación en el sistema de cargos terminaría cuando se unió a una iglesia protestante evangélica en esa misma década. Según su relato, el ciclo ceremonial empezaba el 10 de diciembre cuando se reunían los funcionarios en el poder para decidir quiénes ocuparían los puestos en el año siguiente. Después había una procesión con bailes el 12 de diciembre, día de la Virgen de Guadalupe. ${ }^{4}$ El 24 de diciembre se organizaba una comida y se le daba al alcalde la lista de los nuevos funcionarios, a quienes se mandaba buscar. Al día siguiente, el pueblo bailaba y comía y los viejos funcionarios dejaban su cargo. Don Rosendo menciona que los fiscales organizaban otra comida el día del Año Nuevo, pero no dice mucho más sobre ello. Cabe notar que, en Chicontla, según recuerda don Longino, el cambio de cargos se hacía ese día y no en Navidad. El resto del texto habla de las celebraciones en torno al Carnaval y la Semana Santa, la organización de las cuales habría sido una responsabilidad principal de los fiscales y de las otras personas que tenían un cargo público.

El relato fue grabado por David Beck el 15 de enero de 2003 mediante un SoundScope CD Recorder y un micrófono de condensador de cabeza. La transcripción y la traducción iniciales fueron realizadas en las semanas siguientes por Beck en colaboración con don Rosendo, quien habla bien el español a pesar de dominar mejor el totonaco, y con Longino Barragán Sampayo. Posteriormente, se verificó la transcripción con otros hablantes, en particular con Porfirio Sampayo Macín. La transcripción final se comparó con la grabación original y se modificó según correspondiera en febrero de 2019; también se hicieron las traducciones al inglés en ese momento. La grabación empieza con un breve diálogo en español entre Beck y don Rosendo (sin transcribir) en el cual Beck pregunta sobre los cargos tradicionales y si se cambiaban cada año. Don Rosendo contesta que se cambiaban los funcionarios seis días antes del 16 de diciembre, y después continúa su relato en totonaco. ${ }^{5}$

\footnotetext{
${ }^{4}$ Cabe señalar que, en otro relato sobre estas prácticas que se grabó al año siguiente, don Rosendo indicó que la fecha de la reunión era el 16 de diciembre y asoció el inicio del ciclo de los cargos con las Posadas.

${ }^{5}$ En la traducción inglesa del texto que aparece a continuación, empleamos el término del español cuando no hay una buena palabra correspondiente en inglés. En particular, para pixká:lh preferimos la palabra española fiscal en vez de la palabra inglesa verger y para skújni' usamos regidor en vez de councillor. No encontramos equivalente en inglés para ma:yui:lh, por lo cual adoptamos el término náhuatl topil.
} 


\section{LOS CARGOS}

[NARRADO POR ROSENDO MELO MÁRQUEZ]

(1) ka:ma:sto'hkán ... tza'má ...

ka:-ma:-sto'h-kan tza'má

PL.OBJ-CS-juntos-IDF ese

Los reúnen ... esos ...

They get them together ... those ...

(2) ma:sto'hkán ... [ka:ma:s ... lho: ...] pixká:lh

ma:-sto'h-kan pixká:lh

Cs-juntos-IDF fiscal

Reuinen a los fiscales.

They get the fiscales together.

(3) na'xchík pixká:lh ka:ma:sto'hkán ma:yú:lhnu' he: pixká:lhna', pixka:Ihteniénte nak=i'x-chik pixká:lh ka:-ma:-sto'h-kan ma:yú:lh-nu' he: pixká:lh-na' LOC=3P0-casa fiscal PL.OBJ-CS-juntos-IDF topil-PL y fiscal-PL

pixká:Ih-teniénte

fiscal-teniente

Reúnen en la casa del fiscal a los topiles y a los fiscales, a los fiscales tenientes.

They get the topiles and fiscales, the junior fiscales, together in the house of the fiscal.

(4) entonces, tza'má ...

entonces tza'má

entonces ese

Entonces, ese...

So then, this ...

(5) lhawapalá, tza'má ...

lhawá-palá tza'má

hacer-RPT ese

Lo vuelven a hacer, ese ...

It is done again, this ... 
(6) ti: ja: taá'n a'ntzá:

ti: ja: ta-a'n a'ntzá:

RELH NEG 3PL.SUJ-ir allí

los que no van alli...

those that don't go there ...

(7) tza'má ...

tza'má

ese

ese...

this ...

(8) tza'má ma:he:sto'hni'kán kú'chu'

tza'má ma:-he:-sto'h-ní'-kan kú'chu'

ese cs-espalda-juntos-BEN-IDF alcohol

A esos les bacen juntar refino para ellos.

They are sent to get them cane liquor.

(9) a'htín nakvaso wili:kán tza'má

a'h-tin nak=vaso wilí:-kan tza'má

CLF-uno LOC=vaso poner-IDF ese

Lo ponen en un vaso.

They put that in a glass.

(10) cha:'túnu' ti: ja: tachá:'n tzukukán a'hpu:xte'he:ni'kán i'xkú'chu' a'kxní ja: paláj cha:'n cha:--a'túnu' ti: ja: ta-cha:'n tzukú-kan a'hpu:-xte'hé:-ni'-kan CLF-cada.uno RELH NEG 3PL.SUJ-llegaraallí empezar-IDF coronilla-derramar-BEN-IDF

i'x-kú'chu' a'kxní ja: paláj cha:'n 3PO-alcohol cuando NEG rápido llegarallí

A cada uno que no llega, empiezan a llenarle (el vaso) con refino cuando no llega luego.

For whoever doesn't come, cane liquor is poured for them if they don't come right away. 
(11) entons, [a'kxní ja: ...] a'kxní tachá:'n chuwá: ka:ma:wi:ho:'kán kú'chu' [tu:...] tu: taa'hsto'hni:'tzá'

entonces a'kxní ta-cha:'n chuwá: ka:-ma:-wi:-ho:'-kan kú'chu'

entonces cuando 3PL.SUJ-llegarallí ahora PL.OBJ-CS-comer:CS-TOT-IDF alcohol

tu: $\quad$ ta-a'h-sto'h-ni:'=tzá'

RELLN DCS-cabeza-juntos-PF=ya

Entonces, cuando llegan les hacen tomar todo el refino que se ha juntado.

So then, when they do arrive they are made to drink all the liquor that has accumulated.

(12) ti: xtalawila:nálh xtawate:lhatu'nká' [xlaká'n ...] xlaká'n ja: tu: wa:má

ti: i'x-ta-la-wilá-nan-lh i'x-ta-wa-te:lhá=tu'nká' xlaká'n

RELH PSD-3PL.SUJ-andar-Sentar-ST.PL-PFV PSD-3PL.SUJ-Comer-AMB=muy ellos

ja: tu: wa:má

NEG RELN esto

A los que estaban sentados tomando ... a ellos nada de esto.

For the ones who were sitting there drinking ... they got none of this (treatment).

(13) ja:tzá' tu: ka:'hxte'he:ya:wa:ni'kán porque xlaká'n tawila:nalhtu'nká' tacha:'ní:' a'kxní i'xlá asamblea

ja:=tzá' tu: ka:-a'h-xte'hé:-ya:wá:-ní'-kan porque xlaká'n

NEG=ya RELL PL.OB-cabeza-derramar-pararse-BEN-DF porque ellos

ta-wilá-nan-lh=tu'nká' at a-cha:'n-ni:' a'kxní i'x-la asamblea

3PL.SUJ-sentar-ST.PL-PFV=muy 3PL.SU--llegar.allí-PF cuando 3PO-suyo asamblea

Ya no se los dejan llenos (los vasos) porque ellos están, han llegado a la hora de la asamblea.

They don't set up and fill (their glasses) because they are there, they have come on time to the assembly.

(14) después, [tza'má ...] tza'má a'kxní chuwá: tza'má ...

después tza'má a'kxní chuwá: tza'má

después eso cuando ahora eso

Entonces, ese ... ese cuando abora ese ...

So then, this ... this when, now this ... 
(15) lho:ho:'kán talaksákni'

lhawá-ho:'-kan ta-lak-sak-ni'

hacer-TOT-IDF DCS-INTNS-escoger-NM

Hacen todas las selecciones.

They make all the selections.

(16) ma'htintzá' taha'chi:hó:' [ti: ...] ti: talhawama:'nálh tza'má talaksákni' ho:lu:pixká:lh he: pixka:lhteniénte

ma'h-tin=tzá' ta-ha'chí:-ho:' ti: ta-lhawá-ma:-'nan-lh

CLF-uno=ya 3PL.SU-borracho-TOT RELH 3PL.SU-hacer-PROG-ST.PL-PFV

tza'má ta-lak-sak-ni' ho:lú'-pixká:lh he: pixká:lh-teniénte

eso DCS-INTNS-escoger-NM viejo-fiscal y fiscal-teniente

Una vez que todos están borrachos, los que bacen la selección son el viejo fiscal y los fiscales tenientes.

Once everyone is drunk, the ones who make the selections are the senior fiscal and the junior fiscales.

(17) tama:lhawi:nín tza'má tasto'ho:'kán xli:pó'htu'

$\begin{array}{llll}\text { ta-ma:-lhawá-i-nin } & \text { tza'má } & \text { ta-sto'h-ho:'-kan } & \text { i'x-li:-pó'htu' } \\ \text { 3PL.SU-Cs-hacer-CS-DTRN } & \text { eso } & \text { DCS-juntos-TOT-IDF } & \text { 3P0-GNC-todo }\end{array}$

Mandan que todos se reúnan.

They order everyone to get together.

(18) pixká:Ih he: ma:yú:lhnu' he: tza'má ma:skuju:nu'ní:n, ma'htín tasto'ho:'kán pixká:lh he: ma:yú:lh-nu' he: tza'má ma:-skuj-u:-nú'-ni:n ma'h-tin fiscal y topil-PL $\quad y$ eso CS-trabajar-CS-AGT-PL CLF-uno

ta-sto'h-ho:'-kan

DCS-juntos-TOT-IDF

El fiscal y los topiles y los avisadores, todos se juntan a la vez.

The fiscal and the topiles and the foremen, they all get together at the same time.

(19) [ento ...] a:li:stá:n chuwá: tza'má ...

a:li:stá:n chuwá: tza'má

después ahora eso

Entonces ahora ese ...

So then, now this ... 
(20) a'kxní chin tza'má el doce de diciembre, a'kxní chin tza'má el doce de diciembre cuando llegaraquí eso el doce de diciembre

Cuando llega el doce de diciembre,

When it is December 12,

(21) tza'má, a'kxní taxtutzá' sikwalán,

tza'má a'kxní ta-xtu=tzá' sikwalán

eso cuando DCS-afuera $=$ ya dios

este, cuando sale la Virgen,

that, when the Virgin comes out,

(22) tzukú ma:xtukán tza'má sikwalán, ja:' li:cha:'nkán sikwalán ...

tzukú ma:-xtu-kan tza'má sikwalán ja:' li:-cha:'n-kan sikwalán

empezar CS-afuera-DF eso dios donde INST--legarallí-IDF dios

empiezan a sacar la Virgen, adonde llevan la Virgen ...

they start to take the Virgin (out of the church), (and) where they take the Virgin ...

(23) lhawakán wa:'h

lhawá-kan wa:'h

hacer-IDF tamal

bacen tamales.

they make tamales.

(24) he: taá'n tza'má ... la'haho:lu'nú:n

he: ta-a'n tza'má la'haho:lú'-nu:n

y 3PL.SUJ-ir eso huehue-PL

Yvan los buebues. ${ }^{6}$

And the buebues go around.

(25) u:tzá: ti: ... [wa:má: ...] tasto'ho:'kán na'xchík tza'má [ti: ...] ti: puxku'ma:yú:lh u:tzá: ti: ta-sto'h-ho:'-kan nak=i'x-chik tza'má ti: púxku'-ma:yú:lh eso RELH DCS-juntos-TOT-IDF LOC=3PO-casa eso RELH jefe-topil

Ellos son los que se juntan en la casa del jefe de los topiles.

They are the ones that gather in the house of the chief topil.

6Huehue es palabra náhuatl que significa 'anciano' y se refiere a los personajes enmascarados de la danza tradicional del mismo nombre. 
(26) a'ntzá: tatastó'ha

a'ntzá: ta-ta-sto'h-a

allí 3PL.SU-DCS-juntos-IMPF

Allíse reúnen.

They get together there.

(27) entonces, a'ntzá: lha'ha:nankán

entonces a'ntzá: lha'há:-nan-kan

entonces allí vestir-DTRN-IDF

Entonces, allíse visten.

So then, they get dressed there.

(28) pu'tzakán tza'má vestidos o [...o] tala'hánu:'

pu'tzá-kan tza'má vestidos o ta-la'ha-nu:'

buscar-IDF eso vestidos o DCS-cara-adentro:NM

Buscan los vestidos o las máscaras.

They look for dresses or masks.

(29) pantalún porque la'hma'jtzá' [ja: ... ja: lhú:wa' xhanán ti: ... xha'lhí: pantalún pantalún porque la'hmá'j=tzá' ja: lhú:wa' i'x-hanán pantalones porque hace.mucho=ya NEG muchos PSD-existir

ti: i'x-ha'lhí: pantalún

RELH PSD-tener pantalones

Pantalones, porque hace mucho no eran muchos los que temían pantalones.

Pants, because back then there weren't many (people) who had pants.

(30) wa:' makya:wá' xwili:kaní:'

wa:' makya:wá' i'x-wilí:-kan-ní:'

puros calzón PSD-poner-IDF-PF

Puros calzones ponían.

They wore nothing but calzones. ${ }^{7}$

${ }^{7}$ Calzón se refiere a una "especie de pantalón de manta, amarrado a la cintura y los tobillos" (Diccionario del español de México 2010: 373). 
(31) a:li:stá:n chuwá: pus, tza'má ... a:li:stá:n chuwá: pus tza'má después ahora INTJ eso

Entonces, ahora pues, ese ...

So then, now well, this ...

(32) a'hspúta [tza'má ...] tza'má la posada el veinticuatro ... a'h-sput-a tza'má la posada el veinticuatro cabeza-terminar-IMPF eso la posada el veinticuatro

Termina ... las Posadas, el 24 ...

It is over ... las Posadas, the 24 th ...

(33) tza'má ma:skuju:nu'ní:n [nunu] naka:ma:wí: skujnín tza'má ma:-skuj-u:-nú'-ni:n na-ka:-ma:-wi: skuj-ni'-n eso CS-trabajar-CS-AGT-PL FUT-PL.OBJ-CS-comer:cs trabajar-NM-PL

A los avisadores, los regidores les van a dar de comer. The foremen, the regidores will feed them.

(34) ma'htín a'ntzá: waho:'kán, pu:la:siyájna' he: ma:yú:lhnu' ... ma'h-tin a'ntzá: wa-ho:'-kan pu:la:siyáj-na' he: ma:yú:lh-nu' CLF-uno allí comer-ToT-IDF policía-PL y topil-PL

Comen a la vez todos alli, los policías y los topiles...

They all eat there at once, the policemen and topiles ...

(35) pó'htu' tasto'ho:'kán a'ntzá: tza'má skujnín tzukú naka:ma:ho'tu:kán nakpu:chiwí:n pó'htu' ta-sto'h-ho:'-kan a'ntzá: tza'má skuj-ni'-n tzukú todo DCS-juntos-TOT-IDF allí eso trabajar-NM-PL empezar

na-ka:-ma:-ho't-u:-kan nak=pu:-chiwí:-n FUT-PL.OBJ-CS-tomar-CS-IDF $\quad$ LOC=CTNR-hablar-NM

Todos se reúnen allí los regidores empiezan, se les va a dar de tomar en la presidencia. They all get together there and the regidores will start, they will be given liquor at the town hall.

(36) entonces, tza'má ... ti: xanastankú'nu' ... tza'má ... ma:skuju:nu'ní:n ... entonces tza'má ti: xa-na-stánku'-nu' tza'má ma:-skuj-ui-nú'-ni:n entonces eso RELH DTV-CLIT-hermano.menor-PL eso CS-trabajar-CS-AGT-PL

Entonces, los menores, esos ... los avisadores ... ${ }^{8}$

So then, the junior folk ... the foremen ...

${ }^{8}$ Aquí xanastankú'mu' 'los menores' se refiere a los jóvenes que iban a tener un cargo por primera vez. 
(37) ti: xanapuxkú'nu' talawila:nálh nakchík

ti: $\quad$ xa-na-púxku'-nu' ta-la-wilá-ya:-nan-lh nak=chik

RELH DTV-CLTV-jefe-PL 3 3PL.SUJ-andar-sentar-IMPF-ST.PL-PFV LOC=casa

Los jefes están en casa.

The chiefs are at home.

(38) he: ti: xanastankú'nu' pus, ka:maka:'nkán ka:ma:makaskujni:kán tza'má kú'chu' nakpu:chiwí:n

he: ti: xa-na-stánku'-nu' pus ka:-maka-a'n-kan

y RELH DTV-CLTV-hermano.menor-PL INTJ PL.OBJ-mano-ir-IDF

ka:-ma:-maka-skuj-ni:-kan tza'má kú'chu' nak=pu:-chiwí:-n

PL.OBJ-CS-mano-trabajar-CS-IDF eso alcohol LOC=CTNR-deci-NM

Y a los menores, pues los mandan, los mandan a servir el refino en la presidencia.

And the junior folks, well, they send them, they send them to serve the alcohol in the town hall.

(39) [tza'má ...] ka:maxki:kán [tza'má ...] tza'má skujnín i kú'chu'

ka:-maxkí:-kan tza'má skuj-ni'-n i kú'chu'

PL.OBJ-dar-IDF eso trabajar-NM-PL JF alcohol

A los regidores les dan refino.

The regidores are given liquor.

(40) he: palh wi'x ja: waku'túna' tza'má kú'chu'

he: palh wi'x ja: wa-ku'tún-a' tza'má kú'chu'

y si tú NEG comer-DSD-IMPF:2SG.SUJ eso alcohol

Y si no quieres tomar el refino,

And if you don't want to drink the liquor,

(41) pus, palh hawa'cha'kús i wi'x [ja: ... ja:... ] ja: na: la'hatí:ya' hó'ta pus palh hawá'cha'=kús i wi'x ja: na: la'hatí-ya'

INTJ si muchacho=todavía JF tú NEG todavía gustar.de-IMPF:2SG.SUJ

ho't-a

tomar-IMPF

pues, si eres todavía un muchacho y todavía no te gusta tomar,

well, if you are still a boy and don't like to drink yet, 
(42) kuwésan tama:wa'yán tza'má skujnín ti: tza'jtzá' xaskujnín, ja: wa:má kuwésa=na ta-ma:-wa'yán tza'má skuj-ni'-n ti: tza'j=tzá' a.fuerza=FUT 3PL.SU-CS-comer eso trabajar-NM-PL RELL solamente=ya

xa-skuj-ni'-n ja: wa:má

DTV-trabajar-NM-PL NEG esto

a fuerza los regidores te van a bacer tomar.

the regidores will force you to drink.

(43) a'ntzá: wi'x natzukúya' nahelhsmaní:ya' tza'má kú'chu', nawáya'

a'ntzá: wi'x na-tzukú-ya' na-helh-smaní:-ya'

allí tú FuT-empezar-IMPF:2SG.SUJ FuT-boca-acustombrarse-IMPF:2SG.SUJ

tza'má kú'chu' na-wa-ya'

eso alcohol FUT-comer-IMPF:2SG.SU

Alli te empezarás a acostumbrar a tomar el refino, lo vas a tomar.

That's when you will get used to drinking liquor, you'll drink it.

(44) he: palh wi'x ja: waku'túna' kuwésa natamaxki:yá:n

he: palh wi'x ja: wa-ku'tún-a' kuwésa na-ta-maxkí:-ya:-n

y si tú NEG comer-DSD-IMPF.2SG.SU a.fuerza FuT-3PL.SUJ-dar-IMPF-20BJ

Y si no quieres tomar, a fuerza te van a dar.

And if you don't want to drink, they will force it on you.

(45) tza'má ... natamaxki:ku'tuná:n skujnín

tza'má na-ta-maxkí:-ku'tún-ya:-n skuj-ni'-n

eso FUT-3PL.SUJ-dar-DSD-IMPF-20Bj trabajar-NM-PL

Los regidores van a querer dártelo.

The regidores will want to give it to you.

(46) palh ja: wa' tza'má kú'chu' natama:chi:ni:niná:n i tamaka:'ná:n nakpu:la:chí:n palh ja: wa' tza'má kúchu' na-ta-ma:-chi:-ni:-nin-ya:-n si NEG comer:2SG.SUJ:PFv eso alcohol FUT-3PL.SUJ-CS-amarrar-CS-DTRN-IMPF-20B]

i ta-maka-a'n-ya:-n nak=pu:la:chí:n

JF 3PL.SU-mano-ir-IMPF-20BJ LOC=cárcel

Si no tomas ese refino, van a mandar a encerrarte, te mandan al bote.

If you don't drink the liquor, they will have you locked up, they send you to jail. 
(47) entonces, pus, [tza'má ...] tza'má a:li:stá:n, pus ... entonces pus tza'má a:li:stá:n pus entonces INTJ eso después INTT

Entonces, pues, ese ... ese entonces, pues...

So then, well ... this ... So then, well ...

(48) pus la'hwa:ha'chi:hó:' a'ntzá: skujnín chuwá: a'kxní nala'hchá:'n las cuatro o ... a sí, las cuatro

pus la'hwá:-ha'chí:-ho:' a'ntzá: skuj-ni'-n chuwá: a'kxní

INTJ muchos-borracho-TOT allí trabajar-NM-PL ahora cuando

na-la'h-cha:'n las cuatro 0 a sí las cuatro

FUT-ALTV-llegarallí las cuatro 0 INTJ sí las cuatro

Pues, todos los regidores están bien borrachos cuando ya llegan las cuatro (de la tarde) 0 ... ah sí, las cuatro.

Well, all the regidores are totally drunk when it gets to be four or ... oh yeah, four o'clock.

(49) nala'hchá:'n naa'nkán naka:ta'sati:kán a'ntzá: nata:'wa'yán na'xchík, na'xchík ma:skuju:nú'

na-la'h-cha:'n na-a'n-kan na-ka:-ta'sá-ti:-kan a'ntzá:

FUT-ALTV-llegarallí FUT-ir-IDF FUT-PL.OBJ-llamar-TRN-IDF allí

na-ta:-'wa'yán nak=i'x-chik nak=i'x-chik ma:-skuj-u:-nú'

FUT-CMT-comer LOC=3PO-Casa LOC=3PO-casa CS-trabajar-CS-AGT

Va a llegar la hora, van a ir, los van a llamar, allí los van a invitar a comer en las casas de los avisadores.

(The time) will come, they will go, they will call them, there they will be invited to eat in the houses of the foremen.

(50) entonces, i'xchík ma:skuju:nú' chuwá: [na: ...] na: ka:ma:ho'tu:palakán ka:maxki:kán kú'chu' he: cerveza

entonces i'x-chik ma:-skuj-u:-nú' chuwá: na: ka:-ma:-ho't-ui-palá-kan entonces 3P0-casa Cs-trabajar-CS-AGT ahora todavía PL.0B--CS-tomar-CS-RPT-IDF

ka:-maxkí:-kan kú'chu' he: cerveza

PL.OBj-dar-IDF alcohol y cerveza

Entonces abora también en las casas de los avisadores les hacen tomar otra vez, les dan refino y cerveza.

So then now they also make them drink again in the houses of the foremen, they give them liquor and beer. 
(51) pó'htu' ka:maxki:ho'tkán, tza'má, he: a:li:stá:n ... pó'htu' ka:-maxkí:-ho't-kan tza'má he: a:li:stá:n all PL.OBJ-dar-tomar-IDF eso y después

a todos les dan de tomar, ese, y entonces...

They give everyone something to drink, this, and then ...

(52) chuwá: ka:maxki:kantzá' tza'má lista ti: chu'n tatanu:ní tza'má ... xasá:sti' tza'má ... chuwá: ka:-maxkí:-kan=tzá' tza'má lista ti: chu'=na ta-ta-nu:-ní' ahora PL.0BJ-dar-IDF=ya eso lista RELH PTCL=FUT 3PL.SUJ-DCS-adentro-BEN

tza'má xa-sá:sti' tza'má

eso DTV-nuevo eso

Se les da la lista de los que le van a entrar, los nuevos, los...

They are given the list of those who were going to take office, the new ... the ....

(53) xasá:sti' ma:yú:lh, xasá:sti' ma:skuju:nú', xasá:sti' pixka:lhteniénte xa-sá:sti' ma:yú:lh xa-sá:sti' ma:-skuj-u:-nú' xa-sá:sti' pixka:lh-teniénte DTV-nuevo topil DTV-nuevo cS-trabajar-CS-AGT DTV-nuevo fiscal-teniente los nuevos topiles, los nuevos avisadores, los nuevos fiscales teniente. the new topiles, the new foremen, the new junior fiscales,

(54) maxki:kán lista alhka:lhtéj

maxkí:-kan lista alhka:lhtéj

dar-IDF lista mayor

Se le da la lista al presidente.

The list is given to the mayor.

(55) pus, a:li:stá:n chuwá: tza'má ...

pus a:li:stá:n chuwá: tza'má

INTJ después ahora eso

Pues, entonces ahora, ese ...

Well, so then, now, this ...

(56) tamintzá' nakpu:chiwí:n

ta-min=tzá' nak=pu:-chiwí-n

3PL.SU-venir=ya $\quad$ LOC $=$ CTNR-hablar-NM

Vienen a la presidencia.

They come to the town hall. 
(57) pus ka:maxki:kani:'tzá' tza'má lista, pus ... pus ka:-maxkí:-kan-ni:'=tzá' tza'má lista pus INTJ PL.oB-dar-DDF-PF=ya eso lista INTJ

Se les ba dado la lista, pues...

They have been given the list, well ...

(58) tzukukantzá' ka:wani'kán i ti: chu' nataá'n tza'má li:puxku'ma:skuju:nú' he: ti: naá'n puxku'ma:yú:lh ti: pixka:lhteniénte, ti: chu'n taá'n ...

tzukú-kan=tzá' ka:-wan-ní'-kan i ti: chu' na-ta-a'n tza'má

empezar-IDF=ya $\quad$ PL.OBj-decir-BEN-IDF $\quad$ JF $\quad$ RELH $\quad$ ADM $\quad$ FUT-3PL.SUJ-ir $\quad$ eso

li:-púxku'-ma:-skuj-u:-nú' he: ti: na-a'n púxku'-ma:yú:lh ti:

GNC-jefe-CS-trabajar-CS-AGT y RELH FUT-ir jefe-topil RELH

pixká:llh-teniénte ti: chu' na-ta-a'n

fiscal-teniente RELH PTCL FUT-3PL.SUJ-ir

Se les empieza a decir quién va a ir como el jefe de los avisadores y quién va a ir como jefe de los topiles, quiénes van a ir de fiscal teniente, quiénes van a ir ...

They start to tell them who will be chief foreman and who will be chief topil, who will be a junior fiscal, who will be ...

(59) pus pó'htu' pu:la:siyájna' he: ma:yú:lhnu' he: pixká:lhna', chuwá: ...

pus pó'htu' pu:la:siyáj-na' he: ma:yú:lh-nu' he: pixká:lh-na' chuwá:

INTJ todo policía-PL y topil-PL y fiscal-PL ahora

Pues, todos los policías y los topiles y los fiscales, abora ...

Well, all the policemen and topiles and fiscales, now ...

(60) na: la'hwa:a'nkán ka:pu'tzakán i [ti: ... ] ti:n tatanu:nín

na: la'hwá:-a'n-kan ka:-pu'tzá-kan i ti: na-ta-ta-nu--nin

todavía muchos-ir-DF PL.OBJ-buscar-IDF JF RELH FUT-3PL.SUJ-DCS-adentro-DTRN

También muchos van y buscan a los que van a entrar.

Many also go and look for those who are going to take office. 
(61) entonces, cha:'túnu' chi'n ka:ki:te:te:lhakán i ti: ka:maklate:lhakán ka:le:ma:kánh pu:chiwín

entonces cha:-a'túnu' chi' na-ka:-ki:-tayá-te:lhá-kan i ti:

entonces CLF-cada.uno como FUT-PL.0B-IDV-agarrar-AMB-IDF JF RELH

ka:-maklá-te:lhá-kan ka:-le:n-ma:-kan=nak pu:-chiwí:-n

PL.OBj-encontrar-AMB-IDF PL.OBJ-traer-PROG-IDF=LOC CTNR-decir-NM

Uno por uno los van a ir encontrando, los pasan a traer, a los que van encontrando los llevan a la presidencia.

One by one they will go around finding them, they go to pick them up, those they find they take to the town hall.

(62) ma:panhe:ni'má:ka', ma:panhe:ni'má:ka' xtum tza'má sla'hnamá:ka'

ma:-panh-e:-ni'-ma:-ka' ma:-panh-e:-ní'-ma:-ka' xtum

CS-estallar-CS-BEN-PROG-DDF:PFV CS-estallar-CS-BEN-PROG-IDE:PFV diferente

tza'má sla'h-nan-ma:-ka'

eso tocar-DTRN-PROG-IDE:PFV

Les están echando cohetes, les están echando cohetes, aparte están repicando.

They are setting off rockets for them, they are setting off rockets for them, they are also ringing the (church) bells.

(63) he: xtum kámparas i wili:má:ka' chi' ma:panhe:ni'má:ka', ma:panhe:ni'má:ka'

he: xtum kámpara-s i wilí:-ma:-ka' chi'

y diferente petardo-PL JF poner-PROG-IDF:PFV como

ma:-panh-e:-ní'-ma:-ka' ma:-panh-e:-ní-ma:-ka'

CS-estallar-CS-BEN-PROG-IDF:PFV cS-estallar-CS-BEN-PROG-IDF:PFv

Y aparte están poniendo los petardos, jcómo los están haciendo sonar! jlos están haciendo sonar $!^{9}$

And they are also setting off petards, how they are making them boom! they are making them boom!

(64) a:li:stá:n, ta:'a'mpalakán chuwá: pu'tzakán [ti: ...] ti: spúta, sputakús a:lis:áán ta:-'a'n-palá-kan chuwá: pu'tzá-kan ti: sput-a después CMT-Ir-RPT-IDF ahora buscar-IDF RELH terminar-IMPF

${ }^{9}$ La palabra kámpara 'petardo' se refiere tanto en el totonaco como en el español a un envoltorio de metal que se rellena de pólvora y de tierra compacta, luego se le prende fuego mediante una mecha larga (Beck, 2011: 172). 
sput-a $=$ kús

terminar-IMPF=todavía

Entonces, van juntos otra vez a buscar a los que faltan, todavía faltan.

So then, they go together again to look for those that are missing, still missing.

(65) chi' li:chinte:lhakán cha:'túnu'

chi' li:-chin-te:lhá-kan cha:'-a'túnu'

como InST-llegaraquí-AMB-IDF CLF-cada.uno

Así traen a cada uno.

So they bring each one.

(66) pus, ma:panhe:ni'te:lhakán

pus ma:-panh-e:-ní'-te:lhá-kan

INTJ CS-estallar-CS-BEN-AMB-IDF

Pues, van echando cohetes.

Well, they go along firing off rockets.

(67) pus, kuwésa ma:x tze:waní' xlaká'n i'xtaka'tzí: chi' xtalama:'nálh porque ...

pus kuwésa ma:x tzey-wan-ní' xlakán i'x-ta-ka'tzí: chi'

INTJ a.fuerza DUB bueno-ser-NM ellos PSD-3PL.SUJ-saber como

i'x-ta-la-ma:'-nan-lh porque

PSD-3PL.SUJ-hacer-PROG-ST.PL-PFv porque

Debia de ser bonito, ellos sabian lo que estaban haciendo porque ...

It must have been nice, they knew what they were doing because ...

(68) bueno, na: kit tze:wani' kla'htzílh, porque ...

bueno na: kit tzey-wan-ní i'k-la'htzín-lh porque

bueno todavía yo bueno-ser-NM 1SG.SUJ-ver-PFv porque

Bueno, también yo lo veo bonito porque ...

Well, I also think it was nice because ...

(69) na: kit [ikte: ...] i'kte:servilí: tza'má pu:taskujút

na: kit i'k-te:-servir-la-i: tza'má pu:-ta-skuj-ut

todavía yo 1sG.SUJ-camino-servir-hacer-VBL eso CTNR-DCS-trabajar-NM

también yo serví en un cargo.

I also held a cargo for a time. 
(70) la'hma'jtzá' [chi' ...] chi' i'xlatama:kán la'hmá'j=tzá' chi' i'x-la-tamá:-kan hace.mucho=ya como PSD-hacer-acostar-IDF

Hace mucho así hacían.

They did it like that a long time ago.

(71) a:li:stá:n chuwá: ni la'hcha:'ntzá' el veinticinco, pus ...

a:li:stá:n chuwá: a'kxní la'h-cha:'n==tzá' el veinticinco pus después ahora cuando ALTV-llegarallí=ya el veinticinco INTJ Entonces ahora, viene el 25, pues...

Then now, the 25th comes, well ...

(72) tza'má ...

tza'má

that

ese...

that ...

(73) tza'má naa'nkantzá' naa'hataxtununkán

tza'má na-a'n-kan=tzá' na-a'ha-ta-xtu-nun-kan

eso FUT-ir-IDF=ya FUT-oreja-DCS-afuera-DTRN-IDF

Ellos ya van a ir a entregar sus cargos.

They will go and hand over their cargos.

(74) nale:nkán xchi'xít, xchi'xít káxli' he: i'xchi'xít, a:má: ... he: chuwá: tza'má lukút na-le:n-kan i'x-chi'xít i'x-chi'xít káxli' he: i'x-chi'xít a:má: FUT-agarrar-IDF 3P0-pluma 3P0-pluma pollo y 3P0-pluma esto

chuwá: tza'má lukút ahora eso hueso

Van a llevar las plumas, las plumas de pollo y los huesos.

They will take feathers, chicken feathers and bones.

(75) pó'htu' tu: [tu: ...] lakmasná: tza'má ... tza'má lha'mánh ... pó'htu' tu: lak-mas-na: tza'má tza'má lha'mánh todo RELN APL-pudrirse-ADJ eso eso olla Todo lo que está podrido, esa ... esa olla ...

Everything that is rotten, that ... that pot ... 
(76) katu:wálh tu: xtamojó: naktza'má ... naklha'mánh [nak ..]

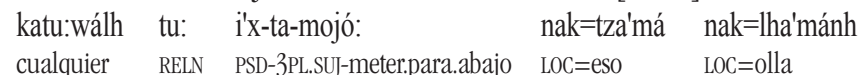
Era cualquier cosa que metían en la olla. It was any old thing that they stuck in the pot.

(77) xtale:ní [ja:, ja:n tanu:ní] ti:n tanu:ni'tzá' li:púxku' ho: ma:skuju:nú' i'xle:ni'palakantzá' i'x-ta-le:n-ní' ti:=na ta-nu:-ni'=tzá' li:-púxku' ho: ma:-skuj-u:-nú' PSD-DCS-agarrar-BEN RELH=FUT DCS-adentro-BEN=ya GNC-jefe $0 \quad$ CS-trabajar-CS-AGT

i'x-le:n-ní-palá-kan=tzá'

PSD-agarrar-BEN-RPT-IDF=ya

Se lo llevaban a los que iban a entrar como jefe o avisador, se lo llevaban otra vez. They took it to the ones who would become chief or foreman, they took it to them again.

(78) chuwá: i'xtalha'ha:nán tza'má cha:'tú', chuwá: i'x-ta-lha'há:-nan tza'má cha:-'tu' ahora PSD-3PL.SUJ-vestir-DTRN eso CLIF-dos Ahora dos personas se vestían. Now two people dressed up.

(79) tza'má ... kristiánus ti: xka:pu'tzakán ti: tza'má ... ti: xtawilí: ha:n ... tza'má kristiánus ti: i'x-ka:-pu'tzá-kan ti: tza'má ti: eso persona RELH PSD-PL.oBj-buscar-IDF RELH eso RELH

i'x-ta-wilí: ha:n PSD-3PL.SU-poner enaguas Estas ... personas a quienes buscaban, las que ponían enaguas ... Those ... people that they looked for, the ones that put on enaguas ...

(80) he: chuwá: cha:'tín [i'x ...] i'xpu'tzakán i ti: xwilí: tza'má vestido he: ... he: chuwá: cha:--tin i'x-pu'tzá-kan i ti: i'x-wilí: tza'má vestido y ahora CLF-uno PSD-buscar-IDF JF RELH PSD-poner eso vestido Y abora buscaban a una persona que ponía vestido, y ... And now they looked for someone to put on a dress, and ... 
(81) he: u:tzá: i'xlé:n pu:kílhku'

he: u:tzá: i'x-le:n pu:kílhku'

y eso PSD-llevar incensario

Y esa (persona) llevaba el incensario.

And that one carried the censer.

(82) tza'má ...

tza'má

that

Ese...

That ...

(83) [i'x ... ] i'xlé:n cha:'tín [ti: ... ti: ... ti: le:má:lh xasiénsu

i'x-le:n cha:-tin ti: le:n-ma:lh xa-siénsu

PSD-llevar CLF-uno RELH agarrar-PROG DTV-incienso

Una persona lo llevaba, la que llevaba el incienso.

A person carried it, the one who is carrying the incense.

(84) cha:'tín xala'haho:lú' mo:joni'te:lhá xasiénsu

cha:-'tin xa-la'haho:lú' mo:jó-ní-te:lhá xa-siénsu

CIF-uno DTV-huehue meter.para.abajo-BEN-AMB DTV-incienso

Uno de los huehues va metiendo el incienso.

One of the huehues goes along adding incense (to the censer).

(85) tza'má pu:kílhku' i'xle:nkán [tza: ...] a'ntzá: ja:' naa'hataxtununkán

tza'má pu:kílhku' i'x-le:n-kan a'ntzá: ja:' na-a'ha-ta-xtu-nun-kan

eso incensario PSD-agarrar-IDF allí donde FUT-oreja-DCS-afuera-DTRN-IDF

El incensario se llevaba allí donde van a entregar los cargos.

The censer was carried there to where they will hand over the cargos.

(86) he: chuwá: ma'hkaujtú' natanu:kán nakchík naki:taxtú ti: tale:ma:'nálh tza'má ha:n he: chuwá: ma'h-kauj-tu' na-ta-nu:-kan nak=chik na-ki-ta-xtu

y ahora CLF-diez-dos FUT-DCS-adentro-IDF LOC=casa FUT-IDV-DCS-afuera

ti: ta-le:n-ma:-nan-lh tza'má ha:n

RELH 3PL.SU-agarrar-PROG-ST.PL-PFV eso enaguas

Y abora los que están llevando enaguas van a entrar y salir de la casa 12 veces. And now those that are wearing enaguas will go in and out of the house 12 times. 
(87) i'xtaki:mo:joni'ma:'nálh tza'má ma:xa'hán i tza'má kú'chu'

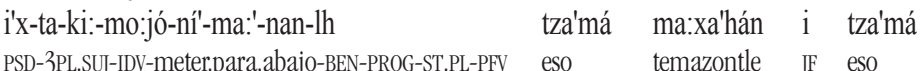

kú'chu'

alcohol

Iban echando refino a los temazontles. ${ }^{10}$

They went along throwing liquor on the stones in the sweatlodge.

(88) a:li:stá:n ... chuwá: naktankilhtín wampaláj ya:palachá [ti: ...] ti: makaskúja tza'má kú'chu'

a:li:stá:n chuwá: nak=tankilhtín wampaláj ya:-palá-chá ti:

después ahora LoC=patio otra.vez pararse-RPT-DIST RELH

maka-skuj-a tza'má kú'chu'

mano-trabajar-IMPF eso alcohol

Entonces, ahora en el patio otra vez está parado el que sirve el refino.

So then, now the one who serves liquor is standing in the patio again.

(89) namaxki:kán, ka:maxki:te:lhakán mat chi' ma'ha'túnu' tatanu:ma:'nálh

na-maxkí:-kan ka:-maxkí:-te:Ihá-kan mat chi' ma'h-a'-túnu'

FUT-dar-IDF PL.0BJ-dar-AMB-IDF CTV como CLF-cada-cada.uno

ta-ta-nu:-ma:'-nan-lh

3PL.SUJ-DCS-adentro-PROG-ST.PL-PFV

Se lo van a dar (refino), se lo van dando así cada vez que están entrando.

They will give them (liquor), they go along giving it to them each time they go inside.

(90) ka:maxki:má:ka' chuwá: tataxtupalá ka:maxki:ma:paláka'

ka:-maxkí-ma:-ka' chuwá: ta-ta-xtu-palá ka:-maxkí-ma:-palá-ka'

PL.0BJ-dar-PROG-IDF:PFv ahora 3PL.SUJ-DCS-afuera-RPT PL.0BJ-dar-PROG-RPT-IDF:PFV

Se lo están dando, abora salen otra vez y se lo están dando otra vez.

They are giving it to them, now they come out and they are giving it to them again.

${ }^{10}$ Ma:xa'bán 'temazontles' son piedras pómez grandes que se calentaban para hacer vapor en el temazcal. 
(91) chu:ntzá: chi' talhi:ma:'nálh

chu:ntzá: chi' ta-lhi:-ma:-nan-lh

así como 3PL.SUJ-bailar-PROG-ST.PL-PFV

Así como están bailando.

They are dancing that way.

(92) he: chu:ntzá:, [tza'má ...] sla'hnama:Ihtzá' mat ti: lhawá, wa:má, violin y guitarra he: chu:ntzá: sla'h-nan-ma:lh=tzá' mat ti: lhawá wa:má violín

y así tocar-DTRN-PROG=ya CTV RELH hacer esto violín

y guitarra

y guitarra

Así, ya están tocando, los que tocan violín y guitarra.

Thus, they are playing now, the ones who play violin and guitar.

(93) tasla'hnama:'nálh

ta-sla'h-nan-ma:-'nan-lh

3PL.SUJ-tocar-DTRN-PROG-ST.PL-PFV

Están tocando.

They are playing.

(94) chuwá: tza'má, chuwá: a:li:stá:n tza'má ...

chuwá: tza'má chuwá: a:li:stá:n tza'má ahora eso ahora después eso

Ahora ese, abora entonces ese ...

Now that, now then, this ...

(95) i'xtataa'hspíta chuwá: naksikwalán chuwá: taa'mpalá [nan ...]

i'x-ta-ta-a'h-spi't-a chuwá: nak=sikwalán ta-a'n-palá

PSD-3PL.SU-DCS-cabeza-voltear-IMPF ahora LOC=iglesia 3PL.SUJ-ir-RPT

Abora regresaban a la iglesia, ahora van otra vez.

Now they returned to the church, now they go again.

(96) taa'mpalá talhí: he: tataxtúlh ma'hkaujtú' na: taki:tanu:palá ma'hkaujtú' tataxtupalá ta-a'n-palá ta-lhi: he: ta-ta-xtu-lh ma'h-kauj-tu' na: 3PL.SU-ir-RPT 3PL.SUJ-bailar y 3PL.SUJ-DCS-afuera-PFV CLF-diez-dos todavía 
ta-ki:-ta-nu:-palá ma'h-kauj-tu' ta-ta-xtu-palá

3PL.SUJ-IDV-DCS-afuera-RPT CLF-diez-dos 3PL.SUJ-DCS-afuera-RPT

Van otra vez a bailar y salen, y también doce veces vuelven a entrar y doce veces vuelven a salir.

They go again to dance and they come out and they go back in twelve times and come back out twelve times.

(97) na:n taki:ma'ha:ma:'nánchi' tza'má kú'chu' he: chu:ntzá: xasiénsu mo:joni'te:lhakán na:=na ta-ki:-ma'há:n-ma:-'nan-chi' tza'má kú'chu' he: chu:ntzá: todavía=FUT 3PL.SUJ-IDV-mandar-PROG-ST.PL-PROX:PFV eso alcohol y así

xa-siénsu mo:jó-ní-te:lhá-kan

DTV-incienso meterpara.abajo-BEN-AMB-IDF

También van a tirar refino allí y así van poniendo incienso.

They will also throw liquor there and they go along adding incense.

(98) a:li:stá:n [ta ...] mat taa'hspúta chuwá: ...

a:li:stá:n mat ta-a'h-sput-a chuwá:

después CTV DCs-cabeza-terminar-IMPF ahora

Entonces, termina abora ...

Then it is over now ...

(99) taa'ntzá' nai'xchík i ti: taxtunumá:lh, pus ...

ta-a'n=tzá' nak=i'x-chik i ti: ta-xtu-nun-ma:lh pus

3PL.SU-ir=ya LOC=3P0-casa JF RELH DCS-afuera-DTRN-PROG INTT

Ya van a la casa del que está saliendo, pues... ${ }^{11}$

Now they go to the house of the one who is leaving his cargo, well ...

(100) tzukukantzá' wapalakana:chá, [wa] wakantzá' tza'má ...

tzukú-kan=tzá' wa-palá-kan-ya:-chá wa-kan=tzá' tza'má

empezar-IDF=ya comer-RPT-IDF-IMPF-DIST comer-IDF=ya eso

Empiezan a comer otra vez allá, comen ese ...

They start to eat there again, they eat the ...

\footnotetext{
${ }^{11}$ El comentario durante la transcripción indica que se trata probablemente de la casa del fiscal mayor saliente.
} 
(101) ja: xma:ntzá' wakán [xa] tza'má xamó:li ja: xma:n=tzá' wa-kan tza'má xa-mó:li NEG solamente=ya comer-IDF eso DTv-mole Ya nomás comen mole.

They eat nothing but mole now.

(102) he: ka:la'hatayakán tza'má [ti: ta ... ] ti: tawilí: ha:n, ti: [ka: ... ka:pu'tzakaní:' he: ka:-la'ha-tayá-kan tza'má ti: ta-wilí: ha:n ti: y PL.OB-cara-agarrar-IDF eso RELH 3PL.SU-poner enaguas RELH

ka:-pu'tzá-kan-ni:' PL.OBJ-buscar-IDF-PF

Y se lo llevan a los que ponen enaguas, a los que han buscado.

And they take it to those who put on enaguas, those they have sought out.

(103) a:li:stá:n chuwá: pus, a'hsputkán a:lisstán chuwá: pus a'h-sput-kan después ahora INTJ cabeza-terminar-IDF Entonces abora, pues, termina. So then, now, well, it is over.

(104) pus, xli:la'halí: chuwá:, pus tza'j ja: xma:ntzá' chuwá: tawá i'xkalhtu'ká'n pus i'x-li:-la'halí: chuwá: pus tza'j ja: xma:n=tzá' chuwá: INTJ 3P0-GNC-mañana ahora INTJ solamente NEG solamente=ya ahora ta-wa i'x-kálhtu'-ka'n 3PL.SUJ-comer 3P0-caldo-PL.P0

Pues, al otro día, pues ... comen abora nada más que caldo.

Well, the next day, well ... they eat nothing but broth.

(105) taho'tho:'wilatzá' tza'má ti: tataxtunuma:'nalhtzá' ta-ho't-ho:'-wilá=tzá' tza'má ti: ta-ta-xtu-nun-ma:-'nan-lh=tzá'
3PL.SU-tomar-TOT-sentar=ya $\quad$ eso $\quad$ RELH $\quad$ 3PL.SU-DCS-afuera-DTRN-PROG-ST.PL-PFV=ya
Ya esos que ya están saliendo ban terminado de tomar.
Those that are leaving office have finished drinking. 
(106) ja: tu: tu: xtale:'htuyún

ja: tu: tu: i'x-ta-lii-a'h-tuyún

NEG RELN RELN PSD-SPL.SUJ-INST-cabeza-preocuparse

Ya no tenían nada de quépreocuparse.

Now they had no more responsibilities.

(107) pus tilhtzá' xtalakwán

pus tilh=tzá' i'x-ta-lak-wan

INTJ dispersado=ya PSD-3PL.SUJ-pierna-decir

Pues, se dispersaron (a sus casas).

Well, they all went (home).

(108) ja:tzá' [wa:má ...] ja:tzá' tatastó'ha a:li:stá:n, porque tanu:ni:'tzá' ti: xasá:sti' tza'má

$\cdots$

ja:=tzá' ja:=tzá' ta-ta-sto'h-a a:li:stá:n porque

$\mathrm{NEG}=\mathrm{ya} \quad \mathrm{NEG}=\mathrm{ya} \quad$ 3PL.SUJ-DCS-cerrado-IMPF después porque

ta-nu:-ni:'=tzá' ti: xa-sá:sti' tza'má

DCS-adentro-PF=ya RELH DTV-nuevo eso

Ya no se juntan entonces porque ya han entrado los nuevos, este ...

They don't get together anymore because now the new officials have started, the ...

(109) ma:skuju:nú' a'ntzá: la'hspúta tza'má

ma:-skuj-u:-nú' a'ntzá: la'h-sput-a tza'má

CS-trabajar-CS-AGT allí INTNs-terminar-IMPF eso

Los avisadores, alli termina eso.

The foremen, it is over (for them) at that point.

(110) chuwá: tza'má ... naki:cha:'mpalá chuwá: a:má: ... Año Nuevo

chuwá: tza'má na-ki--cha:'n-palá chuwá: a:má: año nuevo ahora eso FUT-IDV-llegarallí-RPT ahora eso año nuevo Ahora, va a llegar otra vez el Año Nuevo.

Now, New Year's is going to come again.

(111) ma:wi:nimpalá tza'má ho:lu:pixká:lh [él .. chi'] chi' xli:sputút tza'má ká:'ta' ma:-wi:-nin-palá tza'má ho:lú'-pixká:lh chi' i'x-li-sput-ut tza'má ká:ta' CS-comer:CS-DTRN-RPT eso viejo-fiscal como 3PO-GNC-terminar-NM eso año El viejo fiscal hace una comida otra vez ya que es el fin del año.

The senior fiscal puts on a feast again because it is the end of the year. 
(112) nama:wi:nimpalá chu:ntzá: xlho:palakán, i na: ...

$\begin{array}{lllll}\text { na-ma:-wi:-nin-palá } & \text { chu:ntzá: } & \text { i'x-lhawá-palá-kan } & \text { i } & \text { na: } \\ \text { FUT-CS-comer:CS-DTRN-RPT } & \text { así } & \text { PSD-hacer-RPT-IDF } & \text { JF } & \text { todaviá }\end{array}$

Va a volver a hacer una comida igual a la que hicieron (el 24), todavía... He will hold another feast just like he did before (on the 24th), also ...

(113) na: ma:'haki:ho:'kán tza'má ... xalukút káxli'

na: ma:'hakí:-ho:'-kan tza'má xa-lukút káxli'

todavía levantar-TOT-IDF eso DTv-hueso pollo

También levantan todos los huesos de pollo. ${ }^{12}$

They also gather up all the chicken bones.

(114) xalukút tza'má ... xalukút tza'má ...

xa-lukút tza'má xa-lukút tza'má

DTV-hueso eso DTV-hueso eso

Los huesos de ... los huesos de ...

The bones of ... the bones of ...

(115) xalukút káxli' he: [xa...] tza'má ... i'xchi'xít káxli'

xa-lukút káxli' he: tza'má i'x-chi'xít káxli'

DTV-hueso pollo y eso 3Po-pluma pollo

los huesos de pollo, y las plumas de pollo.

the chicken bones, and the chicken feathers.

(116) he: chuwá: i'xtalé:n tza'má ... [i'x ...] wachí' [i'x ... i'xpa:laká'n i'xtapu'tzá tza'má ...

he: chuwá: i'x-ta-le:n tza'má wachí' i'x-pa:lák-ka'n

y ahora PSD-3PL.SU-llevar eso aparentemente 3P0-bastón-PL.P0

i'x-ta-pu'tzá tza'má

PSD-3PL.SUJ-buscar eso

Y abora les llevaban ese ... parece que ... sus bastones, buscaban ese ...

And now they brought them this ... it seems like ... their staffs, they looked for this ...

${ }^{12}$ Aquí don Rosendo vuelve a contar lo del 24 de diciembre. 
(117) tza'má sé:'hnu' xastalánha' tu: ja: a:'taxkúta tza'má sé:'hnu' xa-stalánha' tu: ja: a:'-ta-xkut-a eso plátano DTV-blanco RELN NEG EXPT-DCS-desatar-IMPF una mata de plátano tierno que no se ba abierto. a young banana plant that still hasn't unfurled.

(118) [i'xtacha:'] i'xtacha:'chi:wilí: [tza'má ... tza'má ...] tza'má xa'ná:t i tu: wani'kán i tza'má nochebuena i'x-ta-cha:--chi:-wilí: $\quad$ tza'má xa'ná:t i tu: wan-ni'-kan PSD-SPL.SUJ-espinilla-amarrar-poner eso flor JF RELN decir-BEN-IDF

i tza'má nochebuena

JF eso nochebuena

Le amarraban (al bastón) esa flor que se llama la nochebuena.

They tied to it (the staff) this flower that they call a "poinsettia".

(119) u:tzá: i'xtachi:wilí: i'xtalé:n tza'má ti: [ti: ... ] chu:nú: xtaá'n tza'má u.tzá: i'x-ta-chi--wilí: i'x-ta-le:n tza'má ti: chu:nú: eso PSD-3PL.SUJ-amarrar-poner PSD-3PL.SUJ-llevar eso RELH así

i'x-ta-a'n tza'má PSD-3PL.SUJ-ir eso

Esa le amarraban (al bastón) y lo llevaban, los que van así, ese ... 13 They tied that to (the staff) and they took it, the ones who go like that, the...

(120) tza'má i'xtaa'hataxtunumpalá a'kxní chuwá: i'xtaa'mpalá tama'hama'hstá: nai'xchík ho:lu:pixká:lh tza'má i'x-ta-a'ha-ta-xtu-nun-palá a'kxní chuwá: i'x-ta-a'n-palá eso PSD-3PL.SUJ-oreja-DCS-afuera-DTRN-RPT cuando ahora PSD-3PL.SUJ-ir-RPT

ta-ma'ha-ma'h-sta: nak=i'x-chik ho:lú'-pixká:lh 3PL.SUJ-mano-AJN-vender LOC=3PO-casa viejo-fiscal

Otra vez volvieron a salir de los cargos, ahora cuando fueron otra vez a entregar los cargos en la casa del viejo fiscal.

They left their cargos again, now when they went again to hand over the cargos at the house of the senior fiscal.

${ }^{13}$ Durante la transcripción don Rosendo explicó que quien llevaba el bastón era el fiscal superior. 
(121) i'xki:la'hpa'hlhma:pi:palakán i tza'má ... pó'htu' tu: i'xtale:mpalá tza'má ho:lu:pixká:Ihna' i'x-ki:-la'h-pa'hlh-ma:pí-palá-kan i tza'má pó'htu' PSD-IDV-INTNS-quebrar-tender-RPT-IDF JF eso todo
tu: $\quad$ i'x-ta-le:n-palá
tza'má ho:lú'-pixká:lh-na'
RELN PSD-3PL.SUJ-AGARRAR-RPT esO viejo-fiscal-PL

Quebraron y dispersaron eso que ... todo lo que volvieron a traer los viejos fiscales.

They broke up and scattered what ... everything that the senior fiscales brought again.

(122) a:li:stá:n chuwá: tza'má ...

a:li:stán chuwá: tza'má

después ahora eso

Entonces ahora ese ...

So then, now, this ...

(123) naa'hsputkán chuwá:, pus ...

$\begin{array}{lll}\text { na-a'h-sput-kan } & \text { chuwá: } & \text { pus } \\ \text { FUT-cabeza-terminar-IDF } & \text { ahora } & \text { INTJ }\end{array}$

Abora van a terminar, pues...

Now they will finish, well ...

(124) pus, tza'má na: ... [tza'má ...] ki:tasu'yupalakán chu'n sikwalán taa'mpalahó:' a'ntzá:

$\begin{array}{llllll}\text { pus } & \text { tza'má } & \text { na: } & \text { ki:-ta-su'yú-palá-kan } & \text { chu'=nak } & \text { sikwalán } \\ \text { INTJ } & \text { eso } & \text { todavía } & \text { IDV-DCS-visible-RPT-IDF } & \text { PTCL=LOC } & \text { church }\end{array}$

ta-a'n-palá-ho:' a'ntzá:

3PL.SUJ-ir-RPT-TOT allí

Pues, ese también ... ese ... vuelven a aparecer en la iglesia, todos van alli otra vez.

Well, also this ... this ... they show up at the church again, they all go there again.

(125) taa'mpalahó:' tama'hama'hsta:palá tataxtupalánh sikwalán

ta-a'n-palá-ho:' ta-ma'ha-ma'h-sta:-palá $\quad$ ta-ta-xtu-palá=nak sikwalán

3PL.SUJ-ir-RPT-TOT 3PL.SUJ-mano-ANN-vender-RPT 3PL.SUJ-DCS-afuera-RPT=LOC iglesia

Todos van otra vez y entregan los cargos y salen de la iglesia otra vez.

They all go there again and hand over their cargos and leave the church again. 
(126) [tza'má ...] taki:lhi:palá taa'mpalá chuwá: [ja:' ...] ja:' nataxtunuma:Ihtzá' tza'má cha: [ti: ...] ti: xaho:lu:pixka:Ihtzá'

ta-ki:-lhi:-palá ta-a'n-palá chuwá: ja:'

3PL.SUJ-IDV-bailar-RPT 3PL.SUJ-ir-RPT ahora donde

na-ta-xtu-nun-ma:lh=tzá' tza'má cha: ti: xa-ho:lú'-pixká:lh=tzá'

FUT-DCS-afuera-DTRN-PROG=ya eso PTCL RELH DTV-viejo-fiscal=ya

Bailan otra vez y van ahora adonde va a salir el que ya es el viejo fiscal.

They dance again and now they go to where the one who is senior fiscal now is going to leave office.

(127) pus, a'ntzá: tzukupalakán ho'tkana:chá, pus, a'ntzá: [tza'má ...] tza'má la'hsputhó:' he: tzejtzá'

a'ntzá: tzukú-palá-kan ho't-kan-ya:-chá a'ntzá: tza'má

allí empezar-RPT-IDF tomar-IDF-IMPF-DIST allí eso

la'h-sput-ho:' he: tzey=tzá'

INTNS-terminar-TOT y bueno=ya

Alli empiezan a tomar otra vez, así ese ... termina todo y ya está bien.

They start to drink there again, and that way ... it's all over and it's good.

(128) tatanu:ni:'tzá' [ti: ...] ti: xasá:sti' tza'má pixká:lhna' ta-ta-nu:-ni:'=tzá' ti: xa-sá:sti' tza'má pixká:lh-na' 3PL.SU-DCS-afuera-PF=ya RELLH DTV-nuevo eso fiscal-PL.

Ya han entrado los nuevos fiscales,

The new fiscales have taken office,

(129) tza'má ho:lu:pixká:lh, tatanu:ni:'tzá'

tza'má ho:lú'-pixká:lh ta-ta-nu:-ni:'=tzá'

eso viejo-fiscal 3PL.SUJ-DCS-adentro-PF=ya

(y) el viejo fiscal, ya ban entrado.

(and) the senior fiscal, they've taken office now.

(130) pus, ja:tzá' taá'n i chuwá: a:li:stá:n xlaká'n, pus ...

pus ja:=tzá' ta-a'n i chuwá: a:lisstán xlakán pus

INTJ NEG=ya 3PL.SUJ-ir JF ahora después ellos INTJ

Ya no van ahora (a reuniones), entonces ellos, pues...

They don't go anymore (to meetings), so then, they, well ... 
(131) a:li:stá:n chuwá:, pus, tza'má ...

a:li:stá:n chuwá: pus tza'má

después ahora INTJ eso

Entonces, ahora pues este...

So then, now well this ...

(132) a'kxní nachimpalá tza'má ... tapa'hsi:nín ...

a'kxní na-chin-palá tza'má tapa'hsi:nín

cuando FuT-llegaraquí-RPT eso carnaval

Cuando va a llegar otra vez el carnaval,

When the time of the carnival is coming,

(133) $[$ a los ... a chi' xle'htú' ma:lhkuyúh [tza'mán ...] tapu'tzá [tza'má ...] tza'má xapu:la'hlho'hó'ho' kí'wi'

chi' i'x-le'h-tu' ma:lhkuyúh ta-pu'tzá tza'má xa-pu:la'h-lho'hó:'-ho'

como 3Po-CLF-dos luna 3PL.SU-buscar eso DTV-adentro-perforado-ADJ

kíwi'

árbol

A como dos meses (antes del carnaval) buscan un palo bueco.

Around two months before (Carnival) they look for a hollow tree.

(134) he: talhawá, wachí' tambor talhawá

he: ta-lhawá wachí' tambor ta-lhawá

y 3PL.SU-hacer aparentemente tambor 3PL.SU-hacer

$Y$ hacen, hacen algo parecido a un tambor.

And they make, they make something like a drum.

(135) chuwá: tapu'tzá tza'má ... talhawá tza'má ...

chuwá: ta-pu'tzá tza'má ta-lhawá tza'má

ahora 3PL.SUJ-buscar eso 3PL.Suj-hacer eso

Abora buscan ese ... hacen ese ...

Now they look for this ... they make this ... 
(136) tapu'tzá tza'má i'xhe:hó'x’a' wa:má ... xkút'i', ho: i'xhe:hó'x'a' katu:wálh animá:lh ta-pu'tzá tza'má i'x-he:-hó'x'a' wa:má xkúti' ho: i'x-he:-hó'x'a' 3PL.Su-buscar eso 3P0-espalda-piel esto coatimundi o 3P0-espalda-piel

katu:wálh animá:lh

cualquier animal

Buscan la piel de este ... tejón o la piel de cualquier animal.

They look for the skin of a ... coatimundi, or the skin of any kind of animal.

(137) tatzukú wa:má ... tarekintalí: wa:'jnanú: wa:má ...

ta-tzukú wa:má ta-requintar-la-i: wa:'jnanú: wa:má

3PL.SUJ-empezar esto 3PL.SUJ-requintar-hacer-VBL allí esto

Empiezan a requintarla alli, este ...

They start to pull it tight, this ...

(138) na'xma'hjú: chi' na'xtuján [tza'má ...] tza'má kí'wi' tu: chi' tali:chi:té: chi' tali:laklhtánka

nak=i'x-ma'hjú: chi' nak=i'x-tuján tza'má kí'wi' tu: chi'

LOC=3PO-fondo como LOC=3Po-pié eso árbol RELN como

ta-li:-chi:-tayá chi' ta-li:-lak-lhta'nk-a

3PL.SUJ-INST-amarrar-agarrar como 3PL.SU-INST-INTNS-requintar-IMPF

en su parte de abajo, como el pie del árbol donde amarran (la piel), la requintaban.

on the bottom, like to the foot of this, this tree that they tie (the skin) to, they tighten it.

(139) a:li:stá:n chuwá: a los ocho, a los quinze días tzukutzá' tama'hasa:'nán [palh ...] palh skawawa'tzá', pus, tza'má ...

a:listá:n chuwá: a los ocho a los quinze días tzukú=tzá'

después ahora a los ocho a los quinze días empezar=ya

ta-ma'ha-sa:'nán palh skawáwa'=tzá' pus tza'má

3PL.SUJ-mano-sonar si seco=ya INTJ eso

Entonces, a los ocho o los quince días empiezan a hacerlo sonar, (a ver) si ya está seco, pues este ...

So then after one or two weeks they begin to percuss it to see if it is dry, well, that ... 
(140) pus a los [me ...] a'kxní a'htín ma:lhkuyúh, pus [ta... ] tala'htzima:'nálh palh i ska:kli'tzá'

$\begin{array}{llllllll}\text { pus a } & \text { los } & \text { a'kxní } & \text { a'h-tin } & \text { ma:lhkuyúh } & \text { pus } & \text { ta-la'htzín-ma:-nan-lh } \\ \text { INTJ } & \text { a } & \text { los } & \text { cuando } & \text { CLF-uno } & \text { luna } & \text { INTJ } & \text { 3PL.SUJ-ver-PROG-ST.PL-PFV }\end{array}$

palh i ska:k-li'=tzá

si JF secar-PFv=ya

Pues, cuando un mes (ya pasó), pues, están viendo si ya se secó.

Well, after a month, well, they are looking to see if it had dried yet.

(141) pus tatzukutzá' tama'hasa:'nante:lhá maka:stín
pus ta-tzukú=tzá' ta-ma'ha-sa:'nán-te:lhá
mak-a:-s-tin

INTJ 3PL.SU-empezar=ya 3PL.SUJ-STM-Sonar-AMB

CLF-ADT-DIM-uno

Pues poco a poco empiezan a ir tocándolo.

Well, little by little they begin to go along playing it.

(142) entonces, a'kxní chuwá: nachín tapa'hsi:nín

\begin{tabular}{|c|c|c|c|}
\hline entonces & a'kxní & chuwá: & na-chin \\
\hline
\end{tabular}

Entonces cuando va a llegar el carnaval,

So then, when it's almost Carnival time,

(143) [ta ...] talé:n [tza'má ...] tza'má tampú:lh [na'x ...] naktza'má ... na'xhé:n pu:sikwalán

ta-le:n tza'má tampú:lh nak=tza'má nak=i'x-he:n pu:sikwalán

3PL.SUJ-llevar eso tambor LOC=eso LOC=3PO-espalda iglesia

llevan ese ... ese tambor en ese ... en el techo de la iglesia.

they take the ... the drum to ... to the roof of the church.

(144) tzukukán ma'hasa:'nanto:lá como que chi' tawán la'hma'jtzá' que ...

tzukú-kan ma'ha-sa:'nán-tawilá como que chi' ta-wan la'hmájj=tzá' que empezar-IDF mano-sonar-sentar como eso como 3PL.SUJ-decir hace.mucho=ya eso Empiezan a sentarse allí tocando, como que, como dicen antes.

They start to sit there drumming, like, like they say long ago. 
(145) wachí' li:ma:tunkuwí:n i [ta ... ta ... ta ...] tali:lhó: tza'má tatzukú ma'hasa:'nán ta:lhmá:n [nak ...] na'xhé:n pu:sikwalán

wachí' li:-ma:-tunkuwí-i:-n i ta-li:-lhawá tza'má

aparentemente INST-CS-amanecer-CS-NM JF $\quad$ 3PL.SUJ-INST-hacer eso

ta-tzukú ma'ha-sa:'nán ta:-lhma:n nak=i'x-he:n pu:sikwalán

3PL.SU-empezar STM-sonar CLIF-largo L.OC=3P0-espalda iglesia

Parece que lo bacen muy de mañana, empiezan a tocar arriba en el techo de la iglesia.

It seems like they do it early in the morning, they start to play up on the roof of the church.

(146) a:li:stá:n nai'xhé:n pu:sikwalán chuwá: ...

a:li:stá:n nak=i'x-he:n pu:sikwalán chuwá:

después LOC=3PO-espalda iglesia ahora

Entonces, en el techo de la iglesia ahora...

So then, on the roof of the church now ...

(147) tinta'ská:'k tama'hakilhwán [tama'ha ...] tatampu:lhnuntawaká

tinta'ská:'k ta-ma'ha-kilh-wan ta-tampú:lh-nun-tawaká

toda.la.noche 3PL.SUJ-mano-boca-decir 3PL.SUJ-tambor-DTRN-arriba

Toda la noche lo hacen sonar con las manos, tamborean alli arriba.

All night they make it sound with their hands, they drum up there.

(148) a:li:stá:n pus tza'má, pus, tayúja chuwá: ... xtum xakú'chu' chi' talé:n tatzukú taho:'to:ká a'ntzá'

a:li:stá:n pus tza'má pus ta-yuj-a chuwá: xtum

después INTJ eso INTJ 3PL.SUJ-irabajo-IMPF ahora diferente

xa-kú'chu' chi' ta-le:n ta-tzukú ta-ho't-tawaká a'ntzá'

DTV-alcohol como 3PL.SU-llevar 3PL.SUJ-empezar 3PL.SUJ-tomar-arriba allí

Entonces pues, ese, pues bajan abora ... no, el refino ya que lo llevan empiezan a tomarlo alli arriba.

So then, well, this, well, they come down now ..., no, the liquor since they bring it they start to drink it up there. 
(149) a:li:stá:n tayúja, pus, tza'má ...

$\begin{array}{llll}\text { a:li:stá:n } & \text { ta-yuj-a } & \text { pus } & \text { tza'má } \\ \text { después } & \text { 3PL.SUj-ir.abajo-IMPF } & \text { INTJ } & \text { eso }\end{array}$

Después bajan, pues, este ...

Then they come down, well, this ...

(150) xli:chuwá: xli:tzi'sajtzá' chuwá: na: natzukukantzá' [na ... ] natasto'hkán

i'x-lii-chuwá: i'x-li:-tzi'sáj=tzá' chuwá: na: na-tzukú-kan=tzá'

3P0-GNC-ahora 3PO-INST-mañana=ya ahora todavía FUT-empezar-IDF=ya

na-ta-sto'h-kan

FUT-DCS-cerrado-IDF

$Y$ hoy, en la mañana, también ahora van a empezar a reunirse.

And today, in the morning, now they also start to get together.

(151) na: tamín xalánh ka:'tziksnán xalánh Cuatechalotla

na: ta-min xalá=nak ka:'tziksnán xalá=nak Cuatechalotla

todavía 3PL.SUJ-venir perteneciente=LOC Tecpatlán perteneciente=LOC Cuatechalotla

También vienen los de Tecpatlán y los de Cuatechalotla.

People from Tecpatlán and Cuatechalotla also come.

(152) xalánh a'hsta'hí:n he: xalánh naktza'má ... Cacahuatlán

xalá=nak a'hsta'hí:n he: xalá=nak nak=tza'má Cacahuatlán

perteneciente $=$ LOC Cuatechalotla y perteneciente $=$ LOC LOC $=$ eso Cacahuatlán

Los de Cuatechalotla y los de ese ... Cacabuatlán,

From Cuatechalotla and from this ... Cacahuatlán,

(153) he: xalá ... li:Chicontla taa'nhó:', taá'n ...

he: xalá li:-Chicontla ta-a'n-ho:' ta-a'n

y perteneciente GNC-Chicontla 3PL.SU-ir-TOT 3PL.SUJ-ir

y todos los de Chicontla, van.

and everyone from Chicontla goes, they go.

(154) tama'hwá tza'má kú'chu' porque ... tza'má tapa'hsi:nín a'kxní xlhawakán ...

ta-ma'h-wa tza'má kú'chu' porque tza'má tapa'hsi:nín

3PL.SUJ-AIN-comer eso alcohol porque eso carnaval

a'kxní i'x-lhawá-kan

cuando PSD-hacer-IDF

Toman el refino porque ... el carnaval, cuando lo bacian ...

They drink liquor because ... the carnival, when they did it ... 
(155) i'xtama:wa:kán, i'xtatama:wá: skujnín a'htú' garrafón tza'má kú'chu' $\begin{array}{llllll}\text { i'x-tama:wá:-kan } & \text { i'x-ta-tama:wá: } & \text { skuj-ni'-n } & \text { a'h-tu' } & \text { garrafón } & \text { tza'má } \\ \text { PSD-comprar-IDF } & \text { PSD-3PL.SU-comprar } & \text { trabajar-NM-PL } & \text { CLF-dos } & \text { garrafón } & \text { eso }\end{array}$

kú'chu'

alcohol

Se compró, los regidores compraban dos garrafones de refino.

It was bought, the regidores bought two demijohns of cane liquor.

(156) he: i'xtatama:wá: pixká:lhna' a'htín garrafón a:ná: xlaká'n i'xtatama:wa:palá he: i'x-ta-tama:wá: pixká:lh-na' a'h-tin garrafón a:-na: xlakán y PSD-3PL.sUJ-comprar fiscal-PL CLF-uno garrafón ADT-todavía ellos

i'x-ta-tama:wá:-palá

PSD-3PL.SUJ-comprar-RPT

Y compraban los fiscales un garrafón y también ellos volvian a comprar otro.

And the fiscales bought a demijohn and they also bought another.

(157) he: ma:skuju:nu'ní:n xtatama:wa:palá chu:ntzá: a:ná: wampaláj xtama:wá: a'htín garrafón

he: ma:-skuj-u:-nú'-ni:n i'x-ta-tama:wá:-palá chu:ntzá: a:-na: wampaláj y CS-trabajar-CS-AGT-PL PSD-3PL.SUJ-comprar-RPT así ADT-todavía otra.vez

i'x-tama:wá: a'h-tin garrafón PSD-comprar CLF-uno garrafón Y los avisadores volvían a comprar uno, así también compraban un garrafón más. And the foremen bought another one, and that way they bought one more demijohn.

(158) entonces, tza'má kú'chu', tza'má ...

entonces tza'má kú'chu' tza'má

entonces eso alcohol eso

Entonces, ese, el refino, ese...

So then, the liquor, the ...

(159) a'kxní i'xtzukukán tasto'hkán [i'x ...] tza'má chi' las diez,

a'kxní i'x-tzukú-kan ta-sto'h-kan tza'má chi' las diez cuando PSD-empezar-IDF DCS-juntos-IDF eso como las diez Cuando empezaron a reunirse como a las diez,

When they started to get together around ten o'clock, 
(160) lakchi:ho:'kani:'tzá' tza'má ... tu: wa:káx wani'kán lak-chi:-ho:'-kan-ni:'=tzá' tza'má tu: wa:káx wan-ní'-kan INTNS-amarrar-TOT-IDF-PF=ya eso RELN vaca decir-BEN-IDF ya han amarrados todos esos que se llamaban toritos. ${ }^{14}$ they have tied together those (things) that they called toritos.

(161) i'xtahe:ma:waká tza'má ... kuxtá:Ih i'xtapu'tzá tza'má xkú:la' $\begin{array}{llllll}\text { i'x-ta-he:-ma:-waká } & \text { tza'má } & \text { kuxtá:lh } & \text { i'x-ta-pu'tzá } & \text { tza'má } & \text { xkú:la' } \\ \text { PSD-3PL.Su-espalda-cs-arriba } & \text { eso } & \text { costal } & \text { PSD-3PL.Su-buscar } & \text { eso } & \text { girasol }\end{array}$ Les ponian costales en las espaldas y buscaban los girasoles. They put sacks on their backs and looked for sunflowers.

(162) i'xtala'hwa:he:xto'howilí: i'x-ta-la'hwá:-he:-xto'ho-wilí: PSD-3PL.SUJ-muchos-espalda-pinchar-poner Les clavaban muchos en las espaldas (de los toritos). They put a lot on the backs (of the toritos).

(163) a:li:stá:n chuwá: pus ... a:listá:n chuwá: pus después ahora INTJ Entonces abora, pues... So then, now, well ...

(164) nale:nkantzá' chuwá: nakpu:i'tá:t [na ...] na-le:n-kan=tzá' chuwá: nak=pu:itá:t FUT-llevar-IDF=ya ahora LOC=centro Van a llevarlos abora al centro.

Now they will take them downtown.

(165) tzukuní' ta'án tza'má ho:lu:pixká:lhna' ta'án tatampu:lhnún tzukú-ní' ta-a'n tza'má ho:lứ-pi:xkálh-na' ta-a'n ta-tampú:lh-nun empezar-BEN 3PL.SUJ-ir eso viejo-fiscal-PL 3PL.SUJ-ir 3PL.SUJ-tambor-DTRN Los viejos fiscales empiezan, van, van tocando el tambor. The senior fiscales start, they go, they go playing the drum.

${ }^{14}$ Un torito es una "figura de carrizo en forma de toro que lleva atados cohetes y otros fuegos artificiales que se encienden mientras un hombre, que lo lleva a cuestas, persigue a los asistentes a la plaza" (Diccionario del español de México 2010: 1602). 
(166) a'kxní tzukukán tampu:lhnunkán mat [natam...]

a'kxní tzukú-kan tampú:lh-nun-kan mat

cuando empezar-IDF tambor-DTRN-IDF CTV

Cuando van a empezar a tocar el tambor, dizque ...

When they start to play the drum, it's said ...

(167) nata:'taxtutzá' tza'má xala'haho:lu'ní:n

na-ta:-'ta-xtu=tzá' tza'má xa-la'haho:lú'-ni:n

FUT-CMT-DCS-afuera=ya eso DTV-huehue-PL

Ya van a salir con los buebues.

Now they will come out with the huehues.

(168) ti:n tza'má ... ti:n taa'hsuyu:nún

ti:=n tza'má ti:=n ta-a'húj-yu:-nun

RELH=JP eso REL RE.H=JF 3PL.SUJ-trampa.de.lazo-TRN-DTRN

Esos que ... que atrapan con lazo.

Those that ... that go lassoing.

(169) entonces, tza'má wa:káxna' ka:la'hwa:ya:wa:kani:'tzá' na'xtankilhtín [tza'má ...] ja:'

i'xhama:nankán, pus ...

entonces tza'má wa:káx-na' ka:-la'hwá:-ya:wá:-kan-ni:'=tzá'

entonces eso vaca-PL PL.OB--muchos-pararse-IDF-PF=ya

nak=i'x-tankilhtín ja:' i'x-hamá:n-nan-kan pus

LOC=3PO-patio donde PSD-jugar-DTRN-IDF INTJ

Entonces, los toritos se ban parado donde jugaban, pues...

So then, the toritos, they have placed them where they played, well ...

(170) ma:ntzá' xtatzukú taa'hpu:nú: kati:wálh kristiánu'

ma:n=tzá' i'x-ta-tzukú ta-a'hpu:-nu: kati:wálh kristiánu'

only=ya PSD-3PL.SUJ-empezar 3PL.SUJ-coronilla-adentro cualquier persona

Cualquier persona nada más empezaba a ponérselos en la cabeza.

And just any old person started to put them on their heads.

(171) [i'x ...] i'xtachá:'n i [ta ... ] taa'hpu:nú: i'xtzukú tali:lhtu'kunún

i'x-ta-cha:'n i ta-a'hpu:-nu: i'x-tzukú

PSD-3PL.SUJ-llegarallí JF 3PL.SUJ-coronilla-adentro PSD-empezar

ta-li:-lhtu'kú-nun

3PL.SUJ-INST-apuñalar-DTRN

Llegaban y se los ponian en la cabeza y empezaban a cornear.

They came and put them on their heads and started goring people with them. 
(172) a:li:stá:n pus, te:lá xtala:li:ma:ma'hawasí:

a:li:stá:n pus te:-la i'x-ta-la:-li:-ma:-ma'ha-was-i:

después INTJ camino-hacer PSD-3PL.SUJ-RCP-INST-CS-STM-resbalar-CS

Entonces, pues, con eso se bacían caer el uno al otro.

So then, they went around knocking each other over with them.

(173) ho: palh liklh tala:li:ma:kilhwakálh ho: palh tala:li:lakalhtu'kúlh

ho: palh liklh ta-la:-li:-ma:-kilh-waká-lh ho: palh

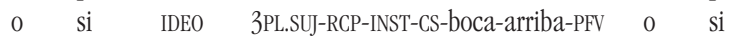

ta-la:-li:-laka-lhtu'kú-lh

3PL.SUJ-RCP-INST-cara-apuñalar-PFv

O se pegan en la boca los unos a los otros o si se pican en la cara.

Or they hit each other smack in the mouth with them or stab each other in the face.

(174) pó'htu' katu:wálh chi' ma:n chi' xtali:lá porque xtaha'chi:tzá' ja:tzá xtala:li:ma'hchuyá: pó'htu' katu:wálh chi' ma:n chi' i'x-ta-li:-la porque todo cualquier como solamente como PSD-3PL.SUJ-INST-hacer porque

i'x-ta-ha'chí:=tzá' ja:=tzá' i'x-ta-la:-li:-ma'h-chuyá:

PSD-3PL.SU-borracho=ya NEG=ya PSD-3PL.SUJ-RCP-INST-ANN-enloquecerse

Cualquier cosa, nada más lo hacían porque estaban borrachos, ya no se respetaban. They did just whatever with them because they were drunk and had no respect for each other.

(175) chi' i'xtahama:nantamá:

chi' i'x-ta-hamá:n-nan-tamá:

como PSD-3PL.SUJ-jugar-DTRN-acostarse

Así como estaban jugando.

That's the way they would play.

(176) a:li:stá:n pus, tza'má kristiánu' ti: xalá ka:'lha:wálhna' ... a:li:stá:n pus tza'má kristiánu' ti: xalá ka:'lha:wálh-na' después INTJ eso persona RELH perteneciente dondequiera-PL Entonces pues, las personas de todas partes...

Well then, people from all over ... 
(177) pus, pó'htu' xka:maxki:kán kú'chu' pus pó'htu' i'x-ka:-maxkí:-kan kú'chu' INTJ todo PSD-PL.OBJ-dar-IDF alcohol Pues, a todos les daban refino.

Well, they gave everyone liquor.

(178) má:ski' puská:n xalánh ka:'tziksnán má:ski' puská:t-n xalá=nak ka:'tziksnán aún mujer-PL pertenecente=LOC Tecpatlán Hasta las mujeres de Tecpatlán. Even the women from Tecpatlán.

(179) ma'htín xtama'hwahó:' kú'chu' ma'h-tin i'x-ta-ma'h-wa-ho:' kú'chu' CLF-uno PSD-3PL.SU--AJN-Comer-TOT alcohol Todos tomaban refino a la vez.

They all drank liquor at the same time.

(180) chi' xtamín taka'tzi:tzá' a'kxní [na ... nalhawakán tza'má ... tapa'hsi:nín chi' i'x-ta-min ta-ka'tzí:=tzá' a'kxní na-lhawá-kan como PSD-3PL.SU-venir 3PL.SU-saber=ya cuando FUT-hacer-IDF

tza'má tapa'hsi:nín eso carnaval

Cómo venían, ya sabian cuándo iban a hacer el carnaval. How they came, they knew when they would celebrate carnival.

(181) pero li:po'htu'tu'nká' i'xtasto'hkán, ja: ti: tza:stín ti: [ja: ...] ja:'x tamína pero li:-pó'htu'=tu'nká' i'x-ta-sto'h-kan ja: ti: tza:stín pero GNC-todo=muy PSD-DCS-close-IDF NEG RELH ni.uno

ti: $\quad$ ja:'=i'x ta-min-a RELH donde=PSD 3PL.SUJ-venir-IMPF

Pero todo el mundo se reunía, no babía ni una persona que no viniera. But everyone got together, there was no one who didn't come.

(182) porque i'xtala'hatí tza'má [chi' ...] chi' ka:'tani:má:ka', chi' ... porque i'x-ta-la'hatí tza'má chi' ka:'ta'ní--ma:-ka' chi' porque PSD-3PL.SUJ-gustar.de eso como celebrar-PROG-DDF:PFv como Porque a ellos les gustaba cómo bacian la fiesta, cómo ... Because they liked the way they were celebrating, like ... 
(183) la'hwa:lá ta:'wamá:ka' kú'chu' he: ... sikáru, pó'htu'

la'hwá:-la ta:--wa-ma:-ka' kú'chu' he: sikáru pó'htu' muchos-hacer cMT-comer-PROG-IDF:PFv alcohol y cigarro todo Muchos se congregan, convidan refino y cigarros, todo.

Many people are there, they give out liquor and cigarettes, everything.

(184) talalha:'wán ti: tamakaskúja xakú'chu' he: [ti: ...] ti: ma:stá: [sas...] xasarí:yu $\begin{array}{lllll}\text { ta-la-lha:'wán } & \text { ti: } & \text { ta-maka-skuj-a } & \text { xa-kú'chu' } & \text { he: } \\ \text { 3PL.SU-hacer-caminar } & \text { RELLH } & \text { 3PL.SUJ-mano-trabajar-IMPF } & \text { DTV-alcohol } & \text { y }\end{array}$

ti: ma:stá: xa-sarí:yu RELH repartir DTV-cerillo Andan los que sirven refino y los que da cerillos.

There is someone serving liquor and someone giving out matches.

(185) entonces, xlaká'n xtali:pa:xuwahó:' xtamína xalánh naka'hsta'hí:n he: xalánh ka:'tziksnán xalánh nakCacahuatlán $\begin{array}{llll}\text { entonces } & \text { xlakán } & \text { i'x-ta--li-pa:xuwá-ho:' } & \text { i'x-ta-min-a } \\ \text { entonces } & \text { ellos } & \text { PSD-3PL.SUJ-INST-alegrarse-TOT } & \text { PSD-3PL.SU-venir-IMPF }\end{array}$ $\begin{array}{lllll}\text { xalá }=\text { nak } & \text { nak=a'hsta'hí:n } & \text { he: } & \text { xalá=nak } & \text { ka:'tziksnán } \\ \text { perteneciente=LOC } & \text { L.OC=Cuatechalótla } & \text { y } & \text { perteneciente=LOC } & \text { Tecpatlán }\end{array}$ xalá=nak nak=Cacahuatlán perteneciente $=$ LOC LOC $=$ Cacahuatlán Entonces, a ellos les daba gusto venir, los de Cuatechalotla, y de Tecpatlán, de Cacabuatlán.

So then, they enjoyed coming, the people from Cuatechalotla, and from Tecpatlán, from Cacahuatlán.

(186) pó'htu' chi' xtasto'hkán li:sla'htu'nká' i'xlatama:kán chi' la'hwa:ka:'tani:má:ka' tapa'hsi:nín

pó'htu' chi' i'x-ta-sto'h-kan li:-sla'h=tu'nká' i'x-la-tamá:-kan chi' todo como PSD-DCS-cerrado-IDF INST-muchos=muy $\quad$ PSD-hacer-acostarse-IDF como

la'hwá:-ka:'ta'ní:-ma:-ka' tapa'hsi:nín muchos-celebrar-PROG-IDF:PFv carnaval Todos, jcómo se juntaba barta gente!, así vivían muchos, muchos venían a celebrar el carnaval.

Everybody, how the people gathered! That's how everybody lived, many people came to celebrate carnival. 
(187) chuwá: a:li:stá:n chuwá: ...

chuwá: a:listá:n chuwá:

ahora después ahora

Abora, entonces, abora ...

Now then, now ...

(188) i'xli:la'halí: wampaláj chu:ntzá: xlhawapalakán, chu:ntzá: i'xle:mpalakán xakú'chu'

i'x-li-la'halí: wampaláj chu:ntzá: i'x-lhawá-palá-kan chu:ntzá:

3P0-GNC-mañana otra.vez así PSD-hacer-RPT-IDF así

i'x-le:n-palá-kan $\quad$ xa-kú'chu'

PSD-agarrar-RPT-IDF DTV-alcohol

El otro día bacian igual de nuevo, asíllevaron otra vez el refino.

The next day they would do the same again, so they brought liquor again.

(189) ja: ma'hstín i spúta tza'má ... tu: wakán i tza'má ... nakpu:i'tá:t porque ka:'tani:má:ka' ja: ma'h-s-tin i sput-a tza'má tu: wa-kan i tza'má

NEG CLF-DIM-Uno JF terminar-IMPF eso REL comer-IDF JF eso

nak=pu:i'tá:t porque ka:'ta'ní:-ma:-ka'

LOC=centro porque celebrar-PROG-IDE:PFv

Nunca les falta qué tomar en el centro porque están baciendo fiesta.

They never lack what to drink downtown because they are celebrating.

(190) ma'htín [tza'j ...] tza'j chu' la:ta:'wama:hó:'ka' kú'chu' má:ski' u:tzá: ti: ma'hatu'nká' lakamiwila:chá

$\begin{array}{llllll}\text { ma'h-tin } & \text { tza'j } & \text { chu' } & \text { la:-ta:-'wa-ma:-ho:'-ka' } & \text { kú'chu' } & \text { má:ski' } \\ \text { CLF-uno } & \text { solamente } & \text { ADM } & \text { RCP-CMT-comer-PROG-TOT-IDF:PFv } & \text { alcohol } & \text { aún }\end{array}$

u:tzá: ti: ma'hát=tu'nká' laka-min-wilá-chá

eso RELH lejos=muy cara-venir-sentar-DIST

A todos no más les convidan el refino basta a los que están mirando desde lejos.

They just give the liquor to everyone, even to those who are watching from far away. 
(191) i'xle:ni'kán ma'htín chi' xma:helhcho'hokán [tza'má ...] tza'má kú'chu', tza'má sikáru i'x-le:n-ní'-kan ma'h-tin chi' i'x-ma:-helhcho'hó-kan tza'má kú'chu' PSD-agarrar-BEN-IDF CLF-uno como PSD-CS-encerrado-IDF eso alcohol

tza'má sikáru

eso cigarro

Les daban refino y cigarros a todos mientras bacían un círculo alrededor.

They gave liquor and cigarettes to everyone as they were standing around in a circle.

(192) pó'htu' xka:maxki:kán ja: ti: ti: ja: xka:maxki:kán

pó'htu' i'x-ka:-maxkí:-kan ja: ti: ti: ja: i'x-ka:-maxkí-kan

todo PSD-PL.OBJ-dar-IDF NEG RELH RELH NEG PSD-PL.OBJ-dar-IDF

A todos se los daban, no babia nadie a quien no se los dieran.

They gave them to everyone, there was no one they didn't give them to.

(193) li:lhu:wa'tu'nká' tasto'hkán, he: ...

li:-lhú:wa'=tu'nká' ta-sto'h-kan he:

GNC-mucho=muy $\quad$ DCS-juntos-IDF $\quad \mathrm{y}$

Muchos se juntan, $y$...

Many people gather and ...

(194) wachí' kit a'kxní s'a'ta'kús xa'kwaní:' ...

wachí' kit a'kxní s'áta'=kús i'x-i'k-wan-ni:'

apparentemente yo cuando pequeño=todavía PSD-1SG.SUJ-ser-PF

Parece que yo, cuando era pequeño ...

It seems like me, when I was young ...

(195) tze:waní kit xa'kla'htzín [chi' ...] chi' i'xlatama:kán

tzey-wan-ní' kit i'x-i'k-la'htzín chi' i'x-la-tamá:-kan

bueno-SER-NM yo PSD-1SG.SUJ-ver como PSD-hacer-acostarse-IDF

lo veía bonito cómo lo bacían.

I thought it was nice, the way they did it.

(196) pó'htu' chuwá: a'kxní chuwá: nachimpalá chuwá: tza'má ... he:ntaxtunún pó'htu' chuwá: a'kxní chuwá: na-chin-palá chuwá: tza'má he:ntaxtunún all ahora cuando ahora FUT-llegar.aquí-RPT ahora eso Semana.Santa Todos ahora, cuando ahora va a llegar otra vez, ahora ese ... Semana Santa.

Everyone now, now when it's coming again now, the ... Holy Week. 
(197) tza'má a'nkán ka:kán tza'má ma'lhú:'k

tza'má a'n-kan ka:-kan tza'má ma'lhú:'k

eso ir-IDF cortar-IDF eso bambú

Van a cortar el tarro. ${ }^{15}$

They go to cut bamboo.

(198) li:tampu:tín i'xa'nkán ka:kán ma'lhú:'k

li:tampu:tín i'x-a'n-kan ka:-kan ma'lhú:'k

los.responsables PSD-ir-IDF cortar-IDF bambú

Todos los que tenían cargos iban a cortar el tarro.

Everyone who held a cargo went to cut bamboo.

(199) a:li:stá:n, i'xli:chinho:'kán ma'lhú:'k ho:tanú:

a:li:stá:n i'x-li:-chin-ho:-'kan ma'lhú:'k ho:tanú:

después PSD-INST-llegar.aquí-TOT-IDF bambú tarde

Entonces todos llegaron con el tarro en la tarde.

So then, everyone came with bamboo in the afternoon.

(200) la'hwa:ma:he:xtimi:kán xcha:'hé:n pu:sikwalán

la'hwá:-ma:-he:-xtim-i:-kan i'x-cha:'hé:n pu:sikwalán

muchos-CS-espalda-parejo-VBL-IDF 3P0-espalda iglesia

Lo amontonan detrás de la iglesia.

They pile it up behind the church.

(201) a:li:stá:n ... tza'má ... na: ...

a:li:stá:n tza'má na:

después eso también

Entonces ... ese ... también ...

So then, ... this ... also ...

(202) a'kxní chuwá: helhtintzá' viernes nawán,

a'kxní chuwá: helh-tin=tzá' viernes na-wan

cuando ahora ClF-uno=ya viernes FuT-be

Ahora cuando va a llegar el Viernes Santo,

Now when Good Friday is coming,

${ }^{15}$ Ma'lhú:'k se refiere a una especie de bambú, posiblemente Guadua aculeata. 
(203) chuwá: la'hwá la:ma:ka'tzini:ho:'kán, tza'má pixká:lhna' pixka:lhtenientená:n chuwá: la'hwá la:-ma:-ka'tzí-ni:-ho:'-kan tza'má pixká:lh-na' ahora muchos RCP-CS-saber-CS-TOT-IDF eso fiscal-PL

pixka:lh-teniénte-na:n

fiscal-teniente-PL

abora a todos los fiscales y los fiscales teniente les avisan. now they tell all the fiscales and junior fiscales.

(204) naa'nkán pu'tzakán li:ta'mpáj

na-a'n-kan pu'tzá-kan li:ta'mpáj

FUT-ir-IDF buscar-IDF palmilla

Van a ir a buscar palmilla.

They will go to look for Chamaedorea palms.

(205) napu'tzakán chu'chó'hx

na-pu'tzá-kan chu'chó'hx

FUT-buscar-IDF flor.de.plátano

Van a buscar la flor de plátano.

They will look for banana flowers.

(206) tza'má ...

tza'má

eso

Ese ...

That ...

(207) entonces, a:chula:tu'nká' ... tza'má la'hma'jtzá' pus ...

entonces a:chulá:=tu'nká' tza'má la'hmá'j=tzá' pus

entonces más=muy eso hace.mucho $=y a$ INTJ

Entonces, mucho más ... este, bace mucho ...

So then, much more ... this, a long time ago ...

(208) i'kla'htziní:' kit [tza'má ...] tza'má lakstín tzu'ma'já:n i [ti: ...] ti: [ja: na: ti: ...] ja: na: ti: i'xa:'ka:skín

i'k-la'htzín-ni:' kit tza'má lakstín tzu'majá:t-n i ti: ja:

1SG.SUJ-ver-PF yo eso niños muchacha-PL JF RELH NEG

na: $\quad$ ti: $\quad$ i'x-a:'-ka:-ski'n

todavía RELH PSD-EXPT-PL.OBJ-pedir

He visto a esas ... a las jóvenes que ... a quienes todavía no pedían.

I've seen the ... the young girls that ... that didn't have fiancés. 
(209) tza'má [i'x ... ] i'xtaán tasto'honún [na ...] naksikwalán tza'má i'x-ta-a'n ta-sto'hó-nun nak=sikwalán eso PSD-3PL.SUJ-ir 3PL.SU-ensartar-DTRN LOC=iglesia Iban a ensartar (flores) en la iglesia.

They went to string (flowers) in the church.

(210) [i'xta ... i'xtalé:n xa'ná:t, tza'má ... [tu: ...] tu: ... tza'má ... ka:na:xa'ná:t wani'kán i'x-ta-le:n xa'ná:t tza'má tu: tu: tza'má ka:na:xa'ná:t PSD-3PL.SU-llevar flor eso RELN RELN eso cempazuchitl

wan-ni'-kan

decir-BEN-IDF

Llevaban flores, esas que se llaman cempazuchitl.

They brought the flowers that they call marigolds.

(211) tza'má la'hwa:la'hsto'honunkán, sla'h tzu'ma'já:n taá'n tasto'honún tza'má la'hwá:-la'h-sto'hó-nun-kan sla'h tzu'ma'já:t-n ta-a'n eso muchos-INTNS-ensartar-DTRN-IDF mucho muchacha-PL 3PL.SUJ-ir

ta-sto'hó-nun

3PL.SUJ-ensartar-DTRN

Muchas ensartan flores, muchas muchachas van a ensartar flores.

Many string flowers, many girls go to string flowers.

(212) i'xtalé:n xa'kska'wi'ká'n [i'x ...]

i'x-ta-le:n i'x-a'kská'wi'-ka'n

PSD-3PL.SUJ-llevar 3P0-canasta-PL.PO

Llevaban sus canastas.

They brought their baskets.

(213) pó'htu' i'xli:xto'honká'n i'xtalé:n, he: chuwá: ...

pó'htu' i'x-li--xto'hó-n-ka'n i'x-ta-le:n he: chuwá:

todo 3PO-INST-pinchar-NM-PL.PO PSD-3PL.SU--llevar y ahora

Todas llevaban sus agujas, y abora ...

They all brought their needles, and now ...

(214) i'xtasto'honunhó:' chuwá: ...

i'x-ta-sto'hó-nun-ho:' chuwá:

PSD-3PL.SUJ-pinchar-DTRN-TOT ahora

Terminaron de ensartar, abora ...

They finished stringing flowers, now ... 
(215) pus, a:má:, tza'má ...

pus a:má: tza'má

INTJ esto eso

Pues, este, ese ...

Well, this, that ...

(216) ma:skuju:nú' ...

ma:-skuj-u:-nú'

CS-trabajar-CS-AGT

Los avisadores ...

The foremen ...

(217) tza'má ... ka:xló: kurús

tza'má ka:x-lhawá kurús

eso arreglado-hacer cruz

arreglan unas cruces.

make some crosses.

(218) [nata ...] taka:xló: kurús ma:skuju:nu'ní:n

ta-ka:x-lhawá kurús ma:-skuj-u:-nú'-ni:n

3PL.suj-arreglado-hacer cruz cS-trabajar-CS-AGT-PL

Los avisadores arreglan unas cruces.

The foremen make some crosses.

(219) naka:ya:wa:kán i [nak ... ] naktza'má ... nakcalle [ja:' ...] ja:' ka:ya:wa:kán

na-ka:-ya:wá:-kan i nak=tza'má nak=calle ja:' ka:-ya:wá:-kan

FUT-PL.OBJ-pararse-IDF JF LOC=eso LOC=calle donde PL.OBJ-parar-IDF

Van a ponerlas en la calle, en la calle las ponen.

They are going to put them in the street, in the street they put them.

(220) a:li:stá:n chuwá: tza'má ... xli:ho:tanú: chuwá: [na ... naka:' ... tza'má ... a:li:stá:n chuwá: tza'má i'x-li:-ho:tanú: chuwá: tza'má después ahora eso 3P0-GNC-tarde ahora eso Entonces, ahora en la tarde, ahora ...

So then, now in the afternoon, now ... 
(221) tza'má ka:ya:wa:kantzá' tza'má ma'lhú:'k [ka: ...] ka:wili:ni'kani:'tzá' tza'má ... li:ta'mpáj he: xa'ná:t tza'má ka:-ya:wá:-kan=tzá' tza'má ma'lhú:'k ka:-wilí--ní-kan-ni:'=tzá' tza'má eso PL.OBJ-pararse-IDF=ya eso bambú PL.oBj-poner-BEN-IDF-PF=ya eso

li:ta'mpáj he: xa'ná:t palmilla y flor Este, ponen el tarro, ya se le han puesto (al tarro) esas palmillas y flores, This, they place the bamboo, they've placed the palms and flowers (on it),

(222) chi' cha:'chi:wakakán

chi' cha:'-chi:-waká-kan

como espinilla-amarrar-arriba-IDF

como están amarradas a lo largo (del tarro).

since they are tied along the length (of the bamboo).

(223) chuwá: tza'má napu'tzapalakán chuwá: tza'má ... tza'má ...

chuwá: tza'má na-pu'tzá-palá-kan chuwá: tza'má tza'má

ahora eso FUT-buscar-RPT-IDF ahora eso eso

Ahora, van a volver a buscar ese, ahora ese ... ese ...

Now, they will go look for it again, now this ... this ...

(224) chu'chó'hx tu: wa:' [ja: ... ja: ... ja: ti: ma'hpe'hxní:'

chu'chó'hx tu: wa:' ja: ti: ma'h-pe'hx-ni:

flor.de.plátano RELN puro NEG RELH cuerpo-pelar-PF

la flor de plátano que nadie ba pelado.

a banana flower that no one has peeled open.

(225) u:tzá: lhtiwilhtiwi xka:chi:wakani'kán na'xhó'sni' tza'má ma'lhú:'k

u:tzá: lhtiwilhtiwi i'x-ka:-chi--waká-ní'-kan nak=i'x-hó'sni' tza'má ma'lhú:'k

eso IDE0 PSD-PL.OBJ-amarrar-arriba-BEN-IDF LOC=3P0-punto eso bambú

Esas las colgaban en la punta del tarro.

These, they hung them on the end of the bamboo.

(226) pus, tze:waní' chi' xlatama:kán la'hma'jtzá'

pus tzey-wan-ní' chi' i'x-la-tamá:-kan la'hmá'j=tzá'

INTJ bueno-ser-NM como PSD-hacer-acostar-IDF hace.mucho=ya

Pues, es bonito cómo lo hacían antes.

Well, it's nice how they did it back then. 
(227) i'kla'htziní:' a'kxní s'a'ta'kús xa'kwaní:', ja: ...

i'k-la'htzín-ni:' a'kxní s'áta'=kús i'x-i'k-wan-ni:' ja:

1SG.SUJ-ver-PF cuando pequeño=todavía PSD-1SG.SUJ-ser-PF NEG

Lo he visto cuando todavía era pequeño, no ...

I saw it when I was still little, no ...

(228) pero ... na: chuwá: ja:tzá' chu:ntzá: chuwá: a:má: lhawakán porque ...

pero na: chuwá: ja:=tzá' chu:ntzá: chuwá: a:má lhawá-kan porque

pero todavía ahora $\mathrm{NEG}=\mathrm{ya}$ así ahora esto hacer-IDF porque

Pero ... abora ya no lo bacen asíporque ...

But ... now they don't do it that way because ...

(229) kuwésa tala'hpali:Ihtzá' [ja: ... ja: ...]

kuwésa ta-la'h-palí:-lh=tzá'

a.fuerza DCS-INTNS-cambiar-PFV=ya

A fuerza ya cambió.

Now it's changed, as it had to.

(230) tali:ma:xanantzá' kristiánu' porque lhu:wa'tzá' pu:Iháj [tanuma:...] tanu:má:chi' $[j a: . .$.

ta-li:-ma:xán-nan=tzá' kristiánu' porque lhú:wa'=tzá' pu:lháj

3PL.SUJ-INST-avergonzar-DTRN=ya persona porque muchos=ya vehículo

ta-nu:-ma:-chi'

DCS-adentro-PROG-PROX:PFV

Ya le da pena a la gente porque vienen muchos carros.

Now people are ashamed because a lot of cars come here.

(231) ja:tzá' ti: lhawá ja:tzá' tu: [tu: ... ] tu: lhawakán [chi' ...] chi' la'hma'jtzá' a'kxní kit i i'ksta'kní:'

ja:=tzá' ti: lhawá ja:=tzá' tu: tu: lhawá-kan chi' la'hmá'j=tzá'

NEG=ya RELH hacer NEG=ya RELN RELN hacer-IDF como hace.mucho=ya

a'kxní kit i i'k-sta'k-ni:'

cuando yo JF 1SG.SUJ-crecer-PF

Y ya nadie lo hace y nada que se hace es como cuando yo crecí.

And now nobody does it and nothing that is done is like it was long ago when I grew up. 
(232) i'kla'htzílh tza'má ... [chi' ...] chi' xlakán

i'k-la'htzín-lh tza'má chi' i'x-la-kan

1SG.SU-ver-PFv eso como PSD-hacer-IDF

Yo vi cómo ... cómo lo hacían.

I saw how ... how they did it.

(233) he: tze:waní' [i'x ...] chi' i'xlhawakán chi' talhawá tza'má la'hma'ha:'ní:n

he: tzey-wan-ní chi' i'x-lhawá-kan chi' ta-lhawá tza'má

y bueno-ser-NM como PSD-hacer-IDF como 3PL.SUJ-hacer eso

la'h-ma'há:'n-ni:n

INTNS-Viejo-PL

Y es bonito cómo se bacía, cómo lo bacían los antiguos.

And it is nice how it was done, how the old-timers did it.

(234) pero ... pero ja: tu: tu: xtama:tzeyi:palá porque lhú:wa' chi' ti: lakamana'kús i $[\mathrm{ti}: \mathrm{x} . ..] \ldots$

pero pero ja: tu: tu: i'x-ta-ma:-tzey-i:-palá porque lhú:wa' chi' pero pero NEG RELN RELN PSD-3PL.SUJ-CS-bueno-CS-RPT porque muchos como

ti: lak-kamá-na'=kús i

RELH APL-niño-PL=todavía JF

Pero ... pero no se ponían de acuerdo porque como muchos eran jóvenes ...

But ... but they couldn't come to an agreement because since many were youths ...

(235) chi' xka:tamaknu:kán tza'má pu:taskujút ...

chi' i'x-ka:-ta-mak-nu:-kan tza'má pu:-ta-skuj-ut

como PSD-PL.OBJ-DCS-cuerpo-adentro-IDF eso CTNR-DCS-trabajar-NM

como los metían en los cargos ...

since they gave them cargos ...

(236) tzukukán ka:ma:ho'tu:kán má:ski' u:tzá: ti: [ ja: na: ...] ja: na: ma'hstín ho'tní:' tzukú-kan ka:-ma:-ho't-u:-kan má:ski' u:tzá: ti: ja: na: ma'h-s-tin empezar-IDF PL.OBJ-CS-tomar-CS-IDF aún eso RELH NEG todavía CLF-DIM-uno

ho't-ni:'

tomar-PF

Empiezan a hacerles tomar, hasta los que nunca han tomado antes.

They start to make them drink, even those that have never drunk before. 
(237) kuwesatu'nká' [i'xtama ... ] i'xtama:wi:nín tza'má skujnín i kú'chu'

kuwésa=tu'nká' i'x-ta-ma:-wa-i:-nin tza'má skuj-ni'-n i kúchu' a.fuerza=muy PSD-3PL.SUJ-CS-Comer-CS-DTRN eso trabajar-NM-PL JF alcohol A fuerza los regidores les hacian tomar refino.

The regidores forced them to drink alcohol.

(238) a:li:stá:n, pus, palh wi'x ja: waku'túna' tza'má kú'chu' ... a:li:stá:n pus palh wi'x ja: wa-ku'tún-a' tza'má kúchu' después INTJ si tú NEG comer-DSD-IMPF:2SG.SUJ eso alcohol Entonces, pues, si tú no quieres tomar el refino ... So, well, if you don't want to drink the alcohol ...

(239) kuwesatu'nká' nataa'hxo'howi:yá:n i ... kuwésa=tu'nká' na-ta-a'hxo'howí-ya:-n i a.fuerza $=$ muy $\quad$ FUT-3PL.SUJ-hacer.tomar-IMPF-20BJ JF a fuerza te van a bacer tomar... they will force you to drink ...

(240) ho: pe:ntú: natali:ma:makawani:yá:n i tza'má skujnín

ho: pe:ntú: na-ta-li:-ma:-maka-wan-ni:-ya:-n i tza'má skuj-ni'-n 0 si.no FUT-3PL.SUJ-INST-CS-mano-decir-CS-IMPF-20Bj JF eso trabajar-NM-PL. si no, los regidores te van a hacer comprar refino a todos. or if not, the regidores will make you buy drinks for everyone.

(241) he: pus como ... wi'x nawáya' porque tza'j natali:me'helhayá:n natawani'yá:n he: pus como wi'x na-wa-ya' porque tza'j y INTJ como tú FUT-comer-IMPF:2SG.SUj porque solamente

na-ta-li:-me'helhá-ya:-n na-ta-wan-ni'-ya:-n FUT-3PL.SUJ-INST-asustar-IMPF-20BJ FUT-3PL.SU-decir-BEN-IMPF-20BJ Ypues como ... tú vas a tomar porque solamente te espantan y te dicen, And well since ... you're going to drink because they just frighten you and tell you,

(242) palh ja: waku'túna' nai'ka:ma:chi:ni:niná:n natawani'yá:n palh ja: wa-ku'tún-a' na-i'k-ka:-ma:-chi:-ni:-nin-ya:-n si NEG comer-DSD-IMPF:2SG.SUJ FUT-1SG.SUJ-PL.OBJ-CS-amarrar-CS-DTRN-IMPF-20BJ

na-ta-wan-ní'-ya:-n FUT-3PL.SUJ-decir-BEN-IMPF-20BJ

"Si no quieres tomar, te voy a encerrar," te van a decir.

"If you don't want to drink, I'm going to lock you up," they are going to tell you. 
(243) he: como wi'x palh s'a'ta'kús

he: como wi'x palh s'áta'=kús

y como tú si pequeño=todavía

Y como tú, si eres joven todavía,

And if you are still young,

(244) pus najikwána' [ja: ...]

pus na-jikwán-a'

INTJ FUT-tener.miedo-IMPF:2SG.SUJ

Pues, vas a tener miedo.

Well, you're going to be afraid.

(245) i'xli:ká:na' tu: kintawani'ma:'nálh porque xlakán [na ...] mat talama:'nálh pu:taskujút, nalakpuwána'

i'x-li:-ká:na' tu: kin-ta-wan-ní-ma:-nan-lh porque xlakán

3PO-GNC-de.veras:NM RELN 10Bj-3PL.SUJ-decir-BEN-PROG-ST.PL-PFV porque ellos

mat ta-la-ma:-nan-lh pu:-ta-skuj-ut

CTV 3PL.SUJ-hacer-PROG-ST.PL-PFv CTNR-DCS-trabajar-NM

na-lak-puwán-a'

FUT-INTNS-pensar-IMPF:2SG.SUJ

"Es verdad lo que me están diciendo porque ellos tienen cargo," vas a pensar.

"It's true what they are telling me because they hold a cargo," you will think.

(246) pus, u:tzá: wi'x [na ... na ... na ...] kuwesatzá'n li:wáya' tza'má kú'chu' pus u:tzá: wi'x kuwésa-tza'=na li:-wa-ya' tza'má kú'chu' INTJ eso tú a.fuerza $=$ ya $=$ FuT INST-comer-2SG.SUj:PFv eso alcohol Pues, por eso nada más a fuerza vas a tomar el refino.

Well, just because of that you're going to drink the liquor under duress.

(247) pero ... sla'h kristiánu' la'hma'jtzá' [ja: ... ja:'x tahó'ta

pero sla'h kristiánu' la'hmá'j=tzá' ja: i'x-ta-ho't-a

pero mucho persona hace.mucho=ya NEG PSD-3PL.SUJ-tomar-IMPF

Pero ... bace mucho mucha gente no tomaba.

But ... long ago many people didn't drink. 
(248) pero a'ntzá: takilhská'tli' tahó'ta tza'má kristiánu' ti: xalakamana'kús ti: [ja: ... ja: na: ...] ja: na:'x tala'hatí tahó'ta

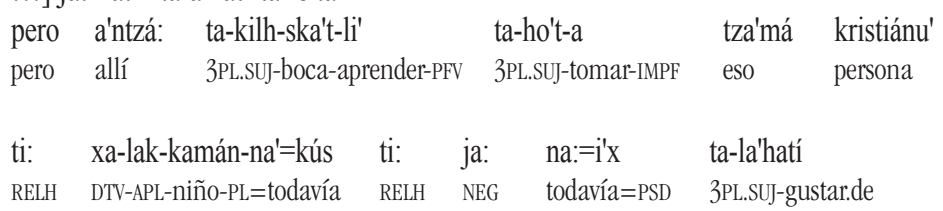

ta-ho't-a

3PL.SUJ-tomar-IMPF

Alli aprenden a tomar las personas que son jóvenes todavía, a quienes todavía no les gustaba tomar.

That's where they learn to drink, people who are still young, people who didn't like to drink yet.

(249) chuwá: tawán tza'má [ti: ... ti: a:chulá: la'hma'ha:'ni:ntzá' tawan chuwá que ja: tu: [tu: ...] tu: talhawaní:' lakamána'

chuwá: ta-wan tza'má ti: a:chulá: la'hma'há:'n-ni:n=tzá' ta-wan ahora 3PL.SUJ-decir eso RELH más anciano-PL=ya 3PL.SUJ-decir

chuwá que ja: tu: tu: ta-lhawá-ni:' lak-kamán-na'

ahora eso NEG RELN RELN 3PL.SUJ-hacer-PF APL-niño-PL

Abora dicen, los más antiguos dicen abora que no les hacian nada a los jóvenes.

Now they say, the oldest old-timers say now that they didn't do anything to the young folk.

(250) pero ... ka:ná: xlaká'n tu: xtalhawama:'nálh ...

pero ka:ná: xlakán tu: i'x-ta-lhawá-ma:'-nan-lh

pero de.veras ellos RELN PSD-3PL.SUJ-hacer-PROG-ST.PL-PFV

Pero, de verás, ellos que estaban haciéndolo ...

But, truly, the ones who were doing it ...

(251) ja: tzey tu: xtalhawama:'nálh porque ...

ja: tzey tu: i'x-ta-lhawá-ma:-nan-lh

NEG bueno RELN PSD-3PL.SUJ-hacer-PROG-ST.PL-PFV

Lo que estaban haciendo no estaba bien porque ...

What they were doing was no good because ... 
(252) lhú:wa' tu: laklhawawilí:lh

lhú:wa' tu: lak-lhawá-wilí--lh

muchos RELN INTNS-hacer-poner-PFV

mucho se arruinó.

a lot of things got wrecked.

(253) talaklhó: [tza'má ...] tza'ma:chá pu:chiwín que chi' xtali:lakli'kxwili:ma:'nálh tza'má ... kuwé:ti'

ta-lak-lhawá tza'má-chá pu:-chiwí-n que chi'

DCS-INTNS-hacer eso-DIST CTNR-decir-NM eso como

i'x-ta-li:-lakli'kx-wilí--ma:-nan-lh tza'má kuwé:ti'

PSD-3PL.SUJ-INST-temblar-poner-PROG-ST.PL-PFv eso cohete

Se dañó la presidencia porque temblaba (por el trueno) de los cohetes. ${ }^{16}$

The town hall was shaken and damaged by (the booming of) the rockets.

(254) tza'má pu:sikwalán chu:ntzá: wampaláj, pus ...

tza'má pu:sikwalán chu:ntzá: wampaláj

eso iglesia así otra.vez

Igual con la iglesia, pues ...

Same for the church, well ...

(255) i'xtama'hawasma:Ihtzá', pero ...

i'x-ta-ma'ha-was-ma:lh=tzá' pero

PSD-DCS-STM-resbalar-PROG=ya pero

Ya se estaba cayendo, pero ...

It was falling down, but ...

(256) ja: ti: ti: wan na'ka:xló: porque ...

ja: ti: ti: wan na-i'k-ka:x-lhawá

NEG RELH RELH decir FUT-1SG.SUJ-arreglado-hacer

Nadie dice, "Yo voy a componerla."

No one says, "I will repair it."

${ }^{16}$ Posteriormente se aclaró que eran las kámparas 'petardos' que dañaron la presidencia. 
(257) [ja: ta ...] ja: tamakawanku'tún kristiánu' nataxo'hó a'htín cooperación tu: chu' nali:ka:xlhawakán tza'má pu:sikwalán

ja: ta-maka-wan-ku'tún kristiánu' na-ta-xo'hó a'h-tin cooperación

NEG 3PL.SUJ-mano-decir-DSD persona FUT-3PL.SU-pagar CLIF-uno donativo

tu: chu' na-li:-ka:x-lhawá-kan tza'má pu:sikwalán

RELN ADM FUT-INST-arreglado-hacer-IDF eso iglesia

No quiere pagar la gente, (no) va a pagar una cooperación con que componer la iglesia.

The people don't want to pay, they (won't) pay a donation to repair the church with.

(258) chu:ntza:tzá' chi' ya:lh

chu:ntzá:=tzá' chi' ya:lh

así=ya como parado

Así como está (la iglesia) abora.

That's how (the church) is now.

(259) ja: ti: [ti: ...] ti: ma'hatzukú naka:xló:

ja: ti: ti: ma'ha-tzukú na-ka:x-lhawá

NEG RELH RELH mano-empezar FUT-arreglado-hacer

Nadie va a empezar a arreglarla.

Nobody will start to fix it.

(260) ja: taxo'honunku'tún kristiánu'

ja: ta-xo'hó-nun-ku'tún kristiánu'

NEG 3PL.SUJ-pagar-DTRN-DSD persona

La gente no quiere pagar.

People don't want to pay.

\section{Abreviaturas}

$\begin{array}{ll}1,2,3 & 1^{\AA}, 2^{\AA}, 3^{\AA} \text { persona } \\ - & \text { linde morfémico } \\ = & \text { clítico } \\ \text { ADJ } & \text { adjetivizador } \\ \text { ADM } & \text { admirativo } \\ \text { ADT } & \text { aditivo } \\ \text { AGT } & \text { agentivo } \\ \text { AJN } & \text { ajeno } \\ \text { ALTV } & \text { aplicativo alativo }\end{array}$




\begin{tabular}{|c|c|}
\hline AMB & ambulante \\
\hline BEN & aplicativo benefactivo \\
\hline CLF & clasificador \\
\hline CLTV & colectivo \\
\hline CMT & comitativo \\
\hline CS & causativo \\
\hline CTNR & contenedor \\
\hline CTV & cuotativo \\
\hline DCS & decausativo \\
\hline DIM & diminutivo \\
\hline DIST & distal \\
\hline DSD & desiderativo \\
\hline DTRN & detransitivo \\
\hline DTV & determinativo \\
\hline DUB & dubitativo \\
\hline EXPT & expectativo \\
\hline FUT & tiempo futuro \\
\hline GNC & genérico \\
\hline IDEO & ideófono \\
\hline IDF & voz indefinida \\
\hline IDV & ida y vuelta \\
\hline IMPF & aspecto imperfectivo \\
\hline INST & aplicativo instrumental \\
\hline INTJ & interjección \\
\hline INTNS & intensificador \\
\hline$J F$ & juntura fonológica \\
\hline LOC & locativo \\
\hline NEG & negativo \\
\hline NM & nominalizador \\
\hline OBJ & objeto \\
\hline PF & aspecto perfecto \\
\hline PFV & aspecto perfectivo \\
\hline PL & plural \\
\hline P0 & posesivo \\
\hline PROG & aspecto progresivo \\
\hline PROX & próximo \\
\hline PSD & tiempo pasado \\
\hline PTCL & partícula \\
\hline RCP & recíproco \\
\hline RELH & relativizador humano \\
\hline RELN & relativizador no humano \\
\hline RPT & repetitivo \\
\hline SG & singular \\
\hline ST.PL & plural del estativo \\
\hline STM & estímulo \\
\hline
\end{tabular}




$\begin{array}{ll}\text { SUJ } & \text { sujeto } \\ \text { TOT } & \text { totalidad } \\ \text { TRN } & \text { transitivizador } \\ \text { VBL } & \text { verbalizador }\end{array}$

\section{Referencias}

BECK, David

2004 Upper Necaxa Totonac. Munich: LINCOM.

2011 Upper Necaxa Totonac Dictionary. Berlin: Mouton.

Cancian, Frank

1965 Economics and Prestige in a Maya Community: The Religious Cargo System in Zinacantan. Stanford, CA: Stanford University Press.

Carrasco, Pedro

1982 Estratificación social en la Mesoamérica prehispánica. Ciudad de México: Secretaría de Educación Pública.

CAulfield, Sueann, Sarah C. Chambers, y Lara PutNAm (eds.)

2005 Honor, Status, and Law in Modern Latin America. Durham: Duke University Press.

Chance, John K., y William B. TAYLoR

1985 "Cofradías and Cargos: An Historical Perspective on the Mesoamerican Civil-religious Hierarchy", American Ethnologist 12: 1-26.

DEWALT, Billie R.

1975 "Changes in the Cargo Systems of Mesoamerica", Anthropological Quarterly 48: 87-105.

Diccionario del español de México

2010 Dirigido por Luis Fernando Lara. Ciudad de México: El Colegio de México.

FRIEDLANDER, Judith

1981 "The Secularization of the Cargo System: An Example from Post-revolutionary Central Mexico" (in Research Reports and Notes), Latin American Research Review 16: 132-143.

Instituto Nacional de Estadística y Geografía (INEGI)

2010 Censo de Población y Vivienda de 2010. http://www.inegi.org.mx/est/contenidos/ proyectos/ccpv/cpv2010/Default.aspx, acceso 21 de diciembre de 2011. 
MASFERRER KAN, Elio Roberto

2006 "Cambio y continuidad entre los totonacos de la Sierra Norte de Puebla". Tesis de maestría, Universidad Iberoamericana, Puebla.

STRESSER-PÉAN, Guy

2009 The Sun-God and the Savior: The Christianization of the Nahua and Totonac in the Sierra Norte de Puebla, Mexico. Boulder, C0: University Press of Colorado.

Recibido: 16 de mayo 2019

Aceptado: 28 de agosto de 2019 
